\title{
Fluid moment and force measurement based on control surface integration
}

\author{
Diana D. Chin ${ }^{1}$ (1) $\cdot$ David Lentink ${ }^{1}$
}

Received: 21 June 2019 / Revised: 3 October 2019 / Accepted: 28 October 2019 / Published online: 3 December 2019

(c) The Author(s) 2019

\begin{abstract}
The moments and torques acting on a deforming body determine its stability and maneuverability. For animals, robots, vehicles, and other deforming objects locomoting in liquid or gaseous fluids, these fluid moments are challenging to accurately measure during unconstrained motion. Particle image velocimetry and aerodynamic force platforms have the potential to resolve this challenge through the use of control surface integration. These measurement techniques have previously been used to recover fluid forces. Here, we show how control surface integration can similarly be used to recover the 3D fluid moments generated about a deforming body's center of mass. We first derive a general formulation that can be applied to any body locomoting in a fluid. We then show when and how this formulation can be greatly simplified without loss of accuracy for conditions commonly encountered during fluid experiments, such as for tests done in wind or water channels. Finally, we provide detailed formulations to show how measurements from an aerodynamic force platform can be used to determine the net instantaneous moments generated by a freely flying body. These formulations also apply more generally to other fluid applications, such as underwater swimming or locomotion over water surfaces.
\end{abstract}

\section{Graphic abstract}

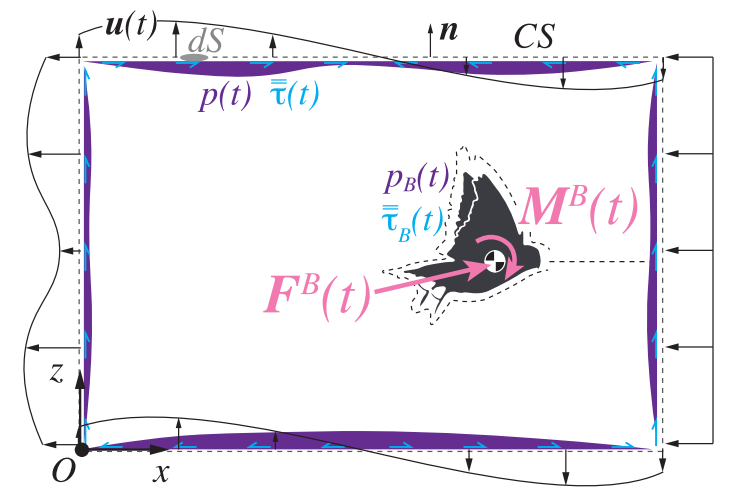

\section{Introduction}

Animals and robots locomoting on the ground, in the water, or in the air all generate forces and moments that dictate their motion. More emphasis is often placed on quantifying forces, which determine the linear accelerations of these

Diana D. Chin

ddchin@alumni.stanford.edu

1 Department of Mechanical Engineering, Stanford University, Stanford, USA deforming bodies, rather than the moments that result in their angular acceleration. However, the moments and torques about a body's center of mass determine the body's stability and maneuverability, making them just as critical to quantify.

The greatest depth of research into locomotion dynamics understandably lies in human biomechanics. While most studies in this field focus on forces and joint moments, several have examined moments and torques about a person's center of mass, especially in sports biomechanics [e.g. longjumps (Ramey 1974), somersaults (Yeadon 1990), high-bar 
dismounts (Hiley and Yeadon 2003), and ski jumps (Arndt et al. 1995)]. These studies often combine the use of experimentally measured kinematics with modeled bodies to quantify forces and moments. To critically improve the accuracy of measured ground reaction forces, several studies have incorporated the use of instrumented trackways (Winter 2009; Zatsiorsky and Zaciorskij 2002). Since the invention of these terrestrial force platforms more than a century ago (Baker 2007), they have played a pivotal role in the analysis of terrestrial locomotion. More recent efforts (Anand and Seipel 2019) have been made towards using these terrestrial platforms to evaluate the ground reaction forces responsible for generating moments about a body's center of mass.

Terrestrial force platforms have also been instrumental in the study of animal and robot locomotion. Many animal studies still rely on combining kinematic measurements with modeled bodies, especially when it comes to studying body moments and torques (Yeadon 1990; Libby et al. 2012). However, through the use of terrestrial force platforms, direct force measurements have now been made for a diverse range of animals, including cockroaches (Full et al. 1991), kangaroo rats (Biewener et al. 1988), chipmunks (Lammers and Zurcher 2011), frogs (Ahn et al. 2004), and horses (Biewener 1998). Similarly, while robot studies often rely on modeling, especially for moment analyses (Libby et al. 2012; Park et al. 2009; Popovic et al. 2005), force platforms (Vukobratovic et al. 2012) or instrumented feet have been used to directly measure forces in legged robots (Sardain and Bessonnet 2004).

Fluid locomotion studies, on the other hand, require a different approach for quantifying the net forces and moments on a body. In studies of human swimming, these alternative approaches have involved people pulling themselves along an instrumented underwater ladder (Toussaint and Vervoorn 1990) or swimming while tethered to a load cell (Morouço et al. 2011). In animal flight studies, tethered locomotion has been used to quantify forces exerted by insects (Lehmann and Dickinson 1998; Sugiura and Dickinson 2009; Dickinson and Götz 1996) and birds (Woike and Gewecke 1978; Marey 1890). Problematically, all of these methods involve constrained locomotion, which does not work well for studying maneuvers that involve body moments and torques. Furthermore, constrained methods are ill-suited for studying deforming bodies, especially because these methods cannot accurately capture inertia effects. Animal welfare concerns aside, imposing constraints may also limit or change an animal's natural movement, especially when significant body deformations are required for generating propulsion.

A popular non-intrusive method for studying fluid locomotion is the use of robotic models. These instrumented robots can be designed to mimic biological motion inside of a flow tank, water or wind tunnel (Georgiades et al. 2009; Triantafyllou et al. 2004; Tan et al. 2007; Dickinson et al.
1999; Lehmann and Pick 2007; Dickson and Dickinson 2004; Bahlman et al. 2013; Hubel and Tropea 2010; Ellington et al. 1996). A potential advantage of using robotic models is that forces generated by different propulsive surfaces can be isolated and compared. These studies again focus primarily on forces, but there have been some studies that have obtained direct measurements of roll, yaw, or pitch moments during animal flight (Cheng et al. 2011; Tucker 2000; Fry et al. 2003). Other non-intrusive methods used to understand the inertial and fluid moments involved during aerial (Dickson et al. 2008; Ennos 1989; Azuma and Watanabe 1988; Lin et al. 2012; Hedrick et al. 2007, 2009; Taylor and Thomas 2002; Dudley 2002) or aquatic locomotion (Lauder and Drucker 2003; Yates 1986) combine measured kinematics with theoretical models and/or computer simulations. However, these models generally require a number of simplifying assumptions; in fish locomotion, for instance, no studies quantify the effects of both the moving body and the fins (Lauder 2010). The force and moment measurements derived in these studies are also difficult to validate directly (Peng and Dabiri 2010).

To actually measure in vivo fluid forces non-intrusively, engineers invented control volume analysis to (simplify and) integrate the Navier-Stokes equations (Vincenti 1982). If the pressure field cannot be measured, then fluid forces and moments can be determined based on velocity and vorticity fields, which are generally determined either computationally or using particle image velocimetry (PIV) (Protas 2007; Howe 1995; Quartapelle and Napolitano 1983; Ragazzo and Tabak 2007; Magnaudet 2011; Wu 1981). Alternatively, the control volume analysis can be simplified by rewriting it into a control surface analysis (Lentink 2018; Rival and van Oudheusden 2017; Wu et al. 2005). Fluid forces can then be recovered by measuring velocity, pressure and shear stress fields on the control surface. The two main experimental implementations of this control surface analysis are highspeed particle image velocimetry (PIV) (Rival and van Oudheusden 2017), and the aerodynamic force platform (AFP) (Lentink 2018). Lentink (2018) recently derived theory to find the conditions under which the control surface formulation of the Navier-Stokes equations can be used to accurately recover fluid forces based on PIV and AFP measurements. PIV involves indirect numerical integration of the measured flow field, while the AFP involves direct mechanical integration of the pressure and shear field via instrumented rigid walls that make up the control surface (Lentink 2018). These recent analyses show how the net fluid force can be measured for freely locomoting animals and robots, but the measurement of the corresponding net fluid moment remains to be addressed.

Here we expand the control surface analysis and derive a new formulation for recovering the fluid moment acting about a body's center of mass. We begin by deriving a general equation based on the conservation of angular momentum, and then 
discuss when and how the formulation can often be simplified for different engineering and scientific applications. As an example, we illustrate how the formulation can be applied to recover the net fluid moment on a bird flying freely inside an aerodynamic force platform, but this example can be generalized to any similarly challenging application of interest in science or engineering.

\section{Control surface formulation}

To analyze fluid moments, we begin by deriving a control volume equation for angular momentum from the general Reynolds transport theorem. We describe the key derivation steps in this section and provide a more detailed derivation in the "Appendix". For an extensive property $\mathbf{N}$ and intensive property $\eta$ of a control mass CM (Vincenti 1982; Sonin 2001):

$$
\begin{aligned}
\frac{\mathrm{d}}{\mathrm{d} t} \iiint_{\mathrm{CM}} \eta \mathrm{d} m= & \iiint_{\mathrm{CV}} \frac{\partial}{\partial t} \eta \rho \mathrm{d} V \\
& +\iint_{\partial V} \eta \rho(\mathbf{u} \cdot \mathbf{n}) \mathrm{d} S=\left[\frac{\mathrm{dN}}{\mathrm{d} t}\right]_{\mathrm{CM}},
\end{aligned}
$$

where $\mathrm{d} m$ is an infinitesimal mass element, $\mathrm{CV}$ is the control volume, $\mathrm{d} V$ is an infinitesimal control volume element, $\partial V$ is the deformable surface of the control volume, and $\mathrm{d} S$ is an infinitesimal control surface element. Additionally, $t$ is time, $\rho$ is fluid density, $\mathbf{u}$ is fluid velocity, and $\mathbf{n}$ is the normal vector of the control surface. We can then select the angular momentum $\mathbf{H}$ about the origin $O$ of the control surface as the extensive property, $\mathbf{N}=\mathbf{H}=\int_{\mathrm{CM}} \mathbf{r} \times \mathbf{u d} m=\int_{\mathrm{CM}}(\mathbf{r} \times \mathbf{u}) \rho \mathrm{d} V$, where $\mathbf{r}$ is the vector connecting the inertial reference point $O$ to the mass element $\mathrm{d} m$. The control volume equation for angular momentum is then:

$\iiint_{\mathrm{CV}} \frac{\partial}{\partial t}(\mathbf{r} \times \mathbf{u}) \rho \mathrm{d} V+\iint_{\partial V}(\mathbf{r} \times \mathbf{u}) \rho(\mathbf{u} \cdot \mathbf{n}) \mathrm{d} S=\left(\frac{\mathrm{d} \mathbf{H}}{\mathrm{d} t}\right)_{\mathrm{CM}}$.

The right-hand side represents external moments resulting from surface forces (pressure $p$ and shear $\tau$ ) and body forces $\mathbf{f}$ acting on the control mass $\left(\mathbf{M}_{\mathrm{CM}}\right)$. In other words, the change in the angular momentum of CM is made up of moments resulting from pressure $\mathbf{M}_{p}$, shear $\mathbf{M}_{\tau}$, and body forces $\mathbf{M}_{\mathrm{f}}$ :

$$
\begin{aligned}
\mathbf{M}_{\mathrm{CM}}= & \left(\frac{\mathrm{d} \mathbf{H}}{\mathrm{d} t}\right)_{\mathrm{CM}} \\
= & \Sigma \mathbf{M}_{\mathrm{ext}}=\mathbf{M}_{p}+\mathbf{M}_{\tau}+\mathbf{M}_{\mathrm{f}} \\
= & -\iint_{\partial V} \mathbf{r} \times p \mathbf{n} \mathrm{d} S \\
& \quad+\iint_{\partial V} \mathbf{r} \times(\overline{\bar{\tau}} \cdot \mathbf{n}) \mathrm{d} S+\iiint_{\mathrm{CV}} \mathbf{r} \times \mathbf{f} \mathrm{d} m .
\end{aligned}
$$

In the most general sense, body forces could also include those due to electric or magnetic fields, but fluid locomotion generally only deals with body forces resulting from gravity. We will, therefore, limit our consideration of body forces to gravity, $\mathbf{f}=\mathbf{g}$. Combining Eqs. (2) and (3) and using $\mathrm{d} m=\rho \mathrm{d} V$, we now have:

$$
\begin{aligned}
\iiint_{\mathrm{CV}} & \frac{\partial}{\partial t}(\mathbf{r} \times \mathbf{u}) \rho \mathrm{d} V+\iint_{\partial V}(\mathbf{r} \times \mathbf{u})(\mathbf{u} \cdot \mathbf{n}) \rho \mathrm{d} S \\
= & -\iint_{\partial V} \mathbf{r} \times p \mathbf{n d} S \\
& +\iint_{\partial V} \mathbf{r} \times(\overline{\bar{\tau}} \cdot \mathbf{n}) \mathrm{d} S+\iiint_{\mathrm{CV}} \mathbf{r} \times \mathbf{g} \rho \mathrm{d} V .
\end{aligned}
$$

We next simplify this expression by introducing the position of the control volume's center of mass $C$ relative to $O, \mathbf{r}^{C / O}$ (Fig. 1a). We note that while a spatial control volume does not have a center of mass, we can treat $\mathrm{CV}$ as a material volume by defining the control volume velocity to be equal to the material velocity at all times (Sonin 2001). As we will discuss in the next section, $\mathbf{r}^{C / O}$ depends on the position of the body (or more specifically, where the volume displaced by the body is) within the $\mathrm{CV}$ and can, therefore, vary in time. The position vector from the origin $O$ to a mass element $\mathrm{d} m$ located at point $P$ can be expressed in terms of $\mathbf{r}^{C / O}$ as $\mathbf{r}=\mathbf{r}^{P / C}+\mathbf{r}^{C / O}$ (where $\mathbf{r}^{P / C}$ is the position vector from $C$ to $\mathrm{d} m)$. By the definition of a center of mass, $\rho \iiint_{\mathrm{CV}} \mathbf{r}^{P / C} \mathrm{~d} V=\iiint_{\mathrm{CV}} \mathbf{r}^{P / C} \mathrm{~d} m=0$. We can thus rewrite the final gravity term as $\rho \iiint_{\mathrm{CV}} \mathbf{r} \times \mathbf{g d} V=\rho \iiint_{\mathrm{CV}}\left(\mathbf{r}^{P / C}+\mathbf{r}^{C / O}\right)$ $\times \mathbf{g d} V=\rho \iiint_{\mathrm{CV}} \mathbf{r}^{C / O} \times \mathbf{g d} V=\mathbf{r}^{C / O} \times \mathbf{g} \rho V$, where $V$ is the fluid volume in the $\mathrm{CV}$. Assuming a constant density flow, Eq. (4) then becomes:

$$
\begin{aligned}
\rho \iiint_{C V} \frac{\partial}{\partial t}(\mathbf{r} \times \mathbf{u}) d V & +\rho \iint_{\partial V}(\mathbf{r} \times \mathbf{u})(\mathbf{u} \cdot \mathbf{n}) d S \\
& =-\iint_{\partial V} \mathbf{r} \times p \mathbf{n} d S+\iint_{\partial V} \mathbf{r} \times(\overline{\bar{\tau}} \cdot \mathbf{n}) d S+\mathbf{r}^{C / O} \times \mathbf{g} \rho V .
\end{aligned}
$$

As detailed in the "Appendix", we can reformulate the lefthand side of Eq. (5) into terms that are more straightforward to evaluate:

$$
\begin{aligned}
\mathbf{M}^{\mathrm{CV} / C}+\mathbf{u}^{C / O} \times \rho \iint_{\partial V} \mathbf{r}(\mathbf{u} \cdot \mathbf{n}) \mathrm{d} S \\
+\mathbf{r}^{C / O} \times\left(-\iint_{\partial V} p \mathbf{n} \mathrm{d} S+\iint_{\partial V}(\overline{\bar{\tau}} \cdot \mathbf{n}) \mathrm{d} S\right. \\
\left.-\rho \iint_{\partial V} \mathbf{u}((\mathbf{u}-\mathbf{v}) \cdot \mathbf{n})\right) \mathrm{d} S \\
=-\iint_{\partial V} \mathbf{r} \times p \mathbf{n} \mathrm{d} S+\iint_{\partial V} \mathbf{r} \times(\overline{\bar{\tau}} \cdot \mathbf{n}) \mathrm{d} S \\
+\mathbf{r}^{C / O} \times \mathbf{g} \rho V,
\end{aligned}
$$


(a)

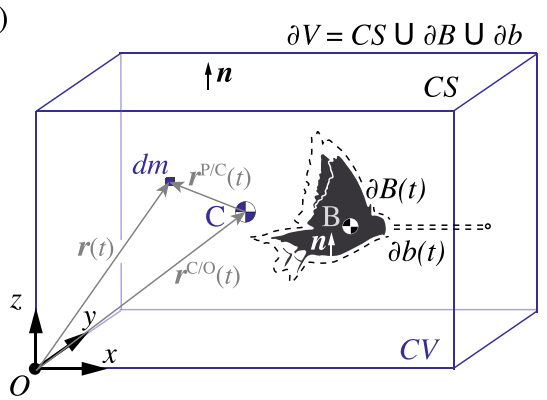

(c)

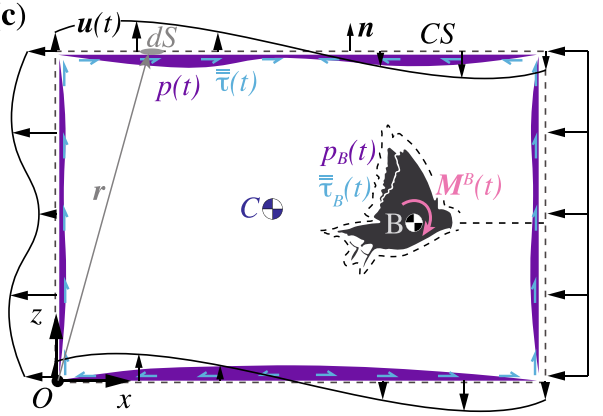

Fig. 1 Control surface diagrams for the fluid moment acting on an arbitrary deforming body. a To derive the control surface formulation for the net fluid moment on the body, we define a control volume CV and control surface $\partial V$ made up of the outer control surface CS, the inner control surface around the deforming body $\partial B$, and an infinitesimal tube $\partial b$ that connects the two, $\partial V=C S \cup \partial B \cup \partial b$, with normal vector $\mathbf{n}$. We define $\mathbf{r}$ as the position vector from the origin $O$ to mass element $\mathrm{d} m$ or a surface element $\mathrm{d} S$, and $\boldsymbol{r}^{C / O}$ is the position vector from $O$ to $C$, the center of mass of the material control volume that coincides with CV. $\boldsymbol{r}^{P / C}$ is the position vector from $C$ to $\mathrm{d} m$. b The net fluid moment on the body about its center of mass $B$ can be calculated as $\boldsymbol{M}^{B}=\boldsymbol{M}^{O}-\boldsymbol{r}^{B / O} \times \mathbf{F}^{B}$ (Mitiguy 2015). $\boldsymbol{M}^{O}$ is the moment

where $\mathbf{M}^{\mathrm{CV} / C}$ is the moment of $\mathrm{CV}$ about its center of mass $C, \mathbf{u}^{C / O}$ is the velocity of $C$ relative to $O$, and $\mathbf{v}$ is the velocity of the control surface.

Next, to isolate fluid moments acting on the body from the rest of the control mass, we follow Lentink (2018) and consider the continuous control surface $\partial V$ in terms of the outer control surface CS, the inner control surface that encloses the deforming body $\partial B$, and a infinitesimally thin tube that connects the body and outer surface $\partial b$, i.e. control surface $(\partial V)=$ outer surface $(\mathrm{CS})+$ tube $(\partial b)+$ body surface $(\partial B)$ (Fig. 1a). Moments on opposite sides of the infinitesimal tube $\partial b$ are equal and opposite and, therefore, cancel out (so all $\partial b$ integrals go to zero). Additionally, the convective term vanishes on the body surface due to the no-flow boundary condition, so the expanded form of Eq. (6) becomes: (b)

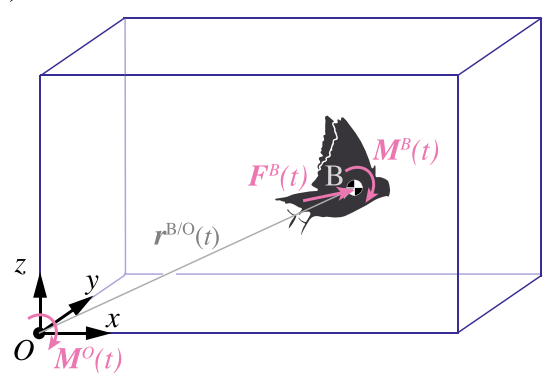

(d)

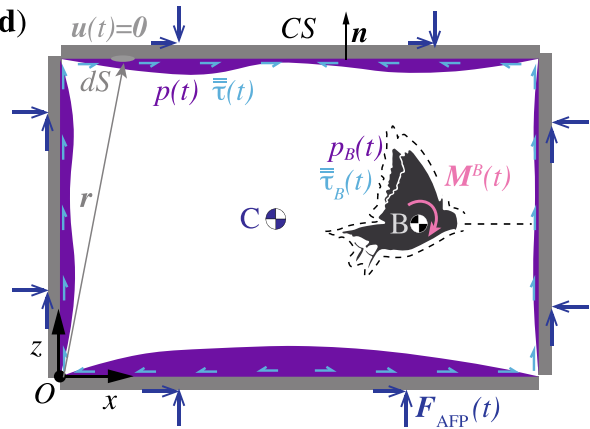

on the body about the origin, $\boldsymbol{r}^{B / O}$ is the position vector of the body's center of mass relative to the origin, and $\boldsymbol{F}^{B}$ is the the net force on the body, which includes net fluid and gravitational forces (Eq. 10). Typical control surface cross sections are shown for c particle image velocimetry and $\mathbf{d}$ an aerodynamic force platform (AFP). Surface pressure $p$ (purple), shear $\overline{\bar{\tau}}$ (light blue), and velocity distributions $\boldsymbol{u}$ are shown on the outer control surfaces (but as in Lentink (2018) not on $\partial B$ and $\partial b$ to avoid clutter). The AFP imposes the no-flow boundary condition $\boldsymbol{u}(t)=0$ on CS via instrumented walls that measure the integrated pressure and shear forces on each wall, $\boldsymbol{F}_{\mathrm{AFP}}$. Diagrams modified from Lentink (2018)

$$
\begin{aligned}
\mathbf{M}^{\mathrm{CV} / C} & +\mathbf{u}^{C / O} \\
& \times \rho\left(\iint_{\mathrm{CS}} \mathbf{r}(\mathbf{u} \cdot \mathbf{n}) \mathrm{d} S+\iint_{\partial B} \mathbf{r}(\mathbf{u} \cdot \mathbf{n}) \mathrm{d} S\right) \\
& +\mathbf{r}^{C / O} \times\left(-\iint_{\mathrm{CS}} p \mathbf{n} \mathrm{d} S+\iint_{\mathrm{CS}}(\overline{\bar{\tau}} \cdot \mathbf{n}) \mathrm{d} S\right. \\
& -\rho \iint_{\mathrm{CS}} \mathbf{u}((\mathbf{u}-\mathbf{v}) \cdot \mathbf{n}) \mathrm{d} S \\
& \left.-\iint_{\partial B} p \mathbf{n} \mathrm{d} S+\iint_{\partial B}(\overline{\bar{\tau}} \cdot \mathbf{n}) \mathrm{d} S\right) \\
= & -\iint_{\mathrm{CS}} \mathbf{r} \times p \mathbf{n} \mathrm{d} S+\iint_{\mathrm{CS}} \mathbf{r} \times(\overline{\bar{\tau}} \cdot \mathbf{n}) \mathrm{d} S \\
& -\iint_{\partial B} \mathbf{r} \times p \mathbf{n} \mathrm{d} S+\iint_{\partial B} \mathbf{r} \times(\overline{\bar{\tau}} \cdot \mathbf{n}) \mathrm{d} S+\mathbf{r}^{C / O} \times \mathbf{g} \rho V .
\end{aligned}
$$


Using this new formulation, we can now derive the total moment acting on the body. The moment caused by pressure and shear on the surface cutout around the body $(\partial B)$ represents the net moment from the body on the fluid. The moment on the body from the fluid will, therefore, be equal and opposite. To find the total external moment on the body with respect to the origin, $\mathbf{M}^{O}$, we must also add in the moment caused by gravity. For a body with mass $m$ and center of mass position $\mathbf{r}^{B / O}$ with respect to the origin (Fig. 1b),

$$
\begin{aligned}
\mathbf{M}^{O}= & -\left(-\iint_{\partial B} \mathbf{r} \times p \mathbf{n d} S+\iint_{\partial B} \mathbf{r} \times(\overline{\bar{\tau}} \cdot \mathbf{n}) \mathrm{d} S\right) \\
& +\mathbf{r}^{B / O} \times m \mathbf{g} .
\end{aligned}
$$

We can solve for $\mathbf{M}^{O}$ using Eq. (7) (see "Appendix" for details) to find:

$$
\begin{aligned}
\mathbf{M}^{O}=- & \mathbf{M}^{\mathrm{CV} / C}-\mathbf{u}^{C / O} \\
& \times \rho\left(\iint_{\mathrm{CS}} \mathbf{r}(\mathbf{u} \cdot \mathbf{n}) \mathrm{d} S+\iint_{\partial B} \mathbf{r}(\mathbf{u} \cdot \mathbf{n}) \mathrm{d} S\right) \\
& -\iint_{\mathrm{CS}}\left(\mathbf{r}-\mathbf{r}^{C / O}\right) \times p \mathbf{n} \mathrm{d} S \\
& +\iint_{\mathrm{CS}}\left(\mathbf{r}-\mathbf{r}^{C / O}\right) \times(\overline{\bar{\tau}} \cdot \mathbf{n}) \mathrm{d} S \\
& +\mathbf{r}^{C / O} \times \rho \iint_{\mathrm{CS}} \mathbf{u}((\mathbf{u}-\mathbf{v}) \cdot \mathbf{n}) \mathrm{d} S \\
& -\mathbf{r}^{C / O} \times(\underbrace{\iint_{\partial B} p \mathbf{n d} S+\iint_{\partial B}(\overline{\bar{\tau}} \cdot \mathbf{n}) \mathrm{d} S}_{\text {Net pressure and shear force from body }}) \\
& +\mathbf{r}^{C / O} \times \mathbf{g} \rho V+\mathbf{r}^{B / O} \times m \mathbf{g} .
\end{aligned}
$$

To further simplify this equation, we replace the body surface pressure and shear integrals by the net force that they have on the fluid in the $\mathrm{CV}$, which is equal and opposite to the net fluid force acting on the body, $\mathbf{F}=-\left(-\iint_{\partial B} p \mathbf{n d} S+\iint_{\partial B}(\overline{\bar{\tau}} \cdot \mathbf{n}) \mathrm{d} S\right)$ :

$$
\begin{aligned}
\mathbf{M}^{O}= & -\mathbf{M}^{\mathrm{CV} / C}-\mathbf{u}^{C / O} \\
& \times \rho\left(\iint_{\mathrm{CS}} \mathbf{r}(\mathbf{u} \cdot \mathbf{n}) \mathrm{d} S+\iint_{\partial B} \mathbf{r}(\mathbf{u} \cdot \mathbf{n}) \mathrm{d} S\right) \\
& -\iint_{\mathrm{CS}}\left(\mathbf{r}-\mathbf{r}^{C / O}\right) \times p \mathbf{n} \mathrm{d} S \\
& +\iint_{\mathrm{CS}}\left(\mathbf{r}-\mathbf{r}^{C / O}\right) \times(\overline{\bar{\tau}} \cdot \mathbf{n}) \mathrm{d} S \\
& +\mathbf{r}^{C / O} \times \rho \iint_{\mathrm{CS}} \mathbf{u}((\mathbf{u}-\mathbf{v}) \cdot \mathbf{n}) \mathrm{d} S \\
& +\mathbf{r}^{C / O} \times \mathbf{F}+\mathbf{r}^{C / O} \times \mathbf{g} \rho V+\mathbf{r}^{B / O} \times m \mathbf{g},
\end{aligned}
$$

where the net fluid force on the body $\mathbf{F}$ is given by (Lentink 2018):

$$
\begin{aligned}
\mathbf{F}= & -\iint_{\mathrm{CS}} p \mathbf{n} \mathrm{d} S+\iint_{\mathrm{CS}}(\overline{\bar{\tau}} \cdot \mathbf{n}) \mathrm{d} S \\
& -\rho \iint_{\mathrm{CS}} \mathbf{u}((\mathbf{u}-\mathbf{v}) \cdot \mathbf{n}) \\
& -\underbrace{\rho \frac{\mathrm{d}}{\mathrm{d} t} \iint_{\partial B} \mathbf{r}(\mathbf{u} \cdot \mathbf{n}) \mathrm{d} S}_{\text {unsteady body force, } \mathbf{U B F}}-\rho \frac{\mathrm{d}}{\mathrm{d} t} \iint_{\mathrm{CS}} \mathbf{r}(\mathbf{u} \cdot \mathbf{n}) \mathrm{d} S .
\end{aligned}
$$

A formulation for the moment on a body about its center of mass $B$, rather than about a theoretical origin, would be more physically meaningful and useful for interpreting the body's rotational dynamics and stability. We, therefore, take one final step to apply the shift theorem for the moment of a set of forces (Mitiguy 2015), which enables us to find the moment on the body about $B$ based on the moment on the body about the origin $\mathbf{M}^{O}$, the position of the body's center of mass relative to the origin $\mathbf{r}^{B / O}$, and the net force on the body $\mathbf{F}^{B}$, which includes the net fluid and gravitational forces on the body (Fig. 1b):

$\mathbf{M}^{B}=\mathbf{M}^{O}-\mathbf{r}^{B / O} \times \mathbf{F}^{B}=\mathbf{M}^{O}-\mathbf{r}^{B / O} \times(\mathbf{F}+m \mathbf{g})$

From Eqs. (8) and (10), we arrive at the general expression for the moment on the body with respect to its center of mass (Fig. 1c):

$$
\begin{aligned}
\mathbf{M}^{B}= & -\mathbf{M}^{C V / C}-\mathbf{u}^{C / O} \times \rho\left(\iint_{C S} \mathbf{r}(\mathbf{u} \cdot \mathbf{n}) d S+\iint_{\partial B} \mathbf{r}(\mathbf{u} \cdot \mathbf{n}) d S\right) \\
& -\iint_{C S}\left(\mathbf{r}-\mathbf{r}^{C / O}\right) \times p \mathbf{n} d S+\iint_{C S}\left(\mathbf{r}-\mathbf{r}^{C / O}\right) \times(\overline{\bar{\tau}} \cdot \mathbf{n}) d S \\
& +\mathbf{r}^{C / O} \times \rho \iint_{C S} \mathbf{u}((\mathbf{u}-\mathbf{v}) \cdot \mathbf{n}) d S \\
& +\left(\mathbf{r}^{C / O}-\mathbf{r}^{B / O}\right) \times \mathbf{F}+\mathbf{r}^{C / O} \times \mathbf{g} \rho V .
\end{aligned}
$$

\section{Results and discussion}

We now examine when the fluid moment formulation (Eq. 11) can be simplified depending on the application and choice of control volume $\mathrm{CV}$. We again define the corresponding control surface $\partial V$ to be made up of the outer control surface CS, the inner control surface around the deforming body $\partial B$, and the infinitesimal tube that connects the two $\partial b$ (Fig. 1a).

\subsection{Non-rotating control volume}

The first term in Eq. (11), the moment of the control volume $\mathrm{CV}$ about its center of mass $\mathbf{M}^{\mathrm{CV} / C}$, can be expressed 
(Mitiguy 2015) in terms of the CV's inertia tensor $\overline{\overline{\mathbf{I}}}^{\mathrm{CV} / \mathrm{C}}$, angular acceleration $\boldsymbol{\alpha}^{\mathrm{CV}}$, angular velocity $\boldsymbol{\omega}^{\mathrm{CV}}$, and angular momentum $\mathbf{H}^{\mathrm{CV} / C}$ about its center of mass $C$ :

$\mathbf{M}^{\mathrm{CV} / C}=\overline{\overline{\mathbf{I}}}^{\mathrm{CV} / C} \cdot \boldsymbol{\alpha}^{\mathrm{CV}}+\boldsymbol{\omega}^{\mathrm{CV}} \times \mathbf{H}^{\mathrm{CV} / \mathrm{C}}$.

We assume in the following analysis that the $\mathrm{CV}$ is nonrotating, $\boldsymbol{\omega}^{\mathrm{CV}}=\mathbf{0}$ and $\boldsymbol{\alpha}^{\mathrm{CV}}=\mathbf{0}$, so $\mathbf{M}^{\mathrm{CV} / C}=\mathbf{0}$. If the $\mathrm{CV}$ does rotate, then this term will need to be measured or derived.

\subsection{Constant body volume and control volume}

Before evaluating the remaining terms in Eq. (11), we will first re-express the position $\mathbf{r}^{C / O}$ and velocity $\mathbf{u}^{C / O}$ of the CV's center of mass in terms of the body's kinematics $\left(\mathbf{r}^{B / O}\right.$, $\mathbf{u}^{B / O}$ ), which are much more straightforward to measure in practice. To do so, we begin by considering a control volume of the same size with no body inside, which will have a fluid mass $m_{V_{0}}$ equal to the sum of the fluid mass displaced by the body $m_{B}$ and the current CV's mass $m_{V}, m_{V_{0}}=m_{B}+m_{V}$. We can then relate the position of the center of mass of the control volume with no body inside, $\mathbf{r}^{C_{\mathrm{o}}}$, to the positions of $B$ and $C$ as

$\mathbf{r}^{C_{\mathrm{o}}} m_{V_{\mathrm{o}}}=\mathbf{r}^{B / O} m_{B}+\mathbf{r}^{C / O} m_{V}$.

For a homogeneous fluid, $\mathbf{r}^{C_{0}}$ will correspond to the geometric center of the control volume. Assuming incompressible flow and neglecting small differences that would result from pressure or temperature variations in the fluid, we can express the masses in Eq. (12) in terms of the fluid density and their corresponding volumes:

$\mathbf{r}^{C_{\mathrm{o}}} \rho V_{\mathrm{o}}=\mathbf{r}^{B / O} \rho V_{B}+\mathbf{r}^{C / O} \rho V$,

where $V_{\mathrm{o}}$ is the CV's volume without the body present, $V_{B}$ is the volume of the fluid displaced by the body, and $V$ is the volume of the actual $\mathrm{CV}$ with the body present (so $\left.V=V_{\mathrm{o}}-V_{B}\right)$. Rearranging, we now have:

$$
\begin{aligned}
\mathbf{r}^{C / O} & =\frac{\mathbf{r}^{C_{\mathrm{o}}} \rho V_{\mathrm{o}}-\mathbf{r}^{B / O} \rho V_{B}}{\rho V_{\mathrm{o}}-\rho V_{B}}=\frac{\mathbf{r}^{C_{\mathrm{o}}} V_{\mathrm{o}}-\mathbf{r}^{B / O} V_{B}}{V_{\mathrm{o}}-V_{B}} \\
& =\frac{V_{\mathrm{o}}}{V} \mathbf{r}^{C_{\mathrm{o}}}-\frac{V_{B}}{V} \mathbf{r}^{B / O} .
\end{aligned}
$$

If we additionally assume that the volume of the body and $\mathrm{CV}$ remain constant,

$\mathbf{u}^{C / O}=\frac{V_{\mathrm{o}}}{V} \mathbf{u}^{C_{\mathrm{o}} / O}-\frac{V_{B}}{V} \mathbf{u}^{B / O}$,

where $\mathbf{u}^{C_{0}} / O$ is the velocity of the CV's center of mass (relative to the origin $O$ ) without the body present. A constant body volume is generally a safe assumption for most fluid experiments, but we will consider the case when $V_{B}$ is variable in Sect. 3.7. Next, if the outer control surface CS is constant, or only deforms in such a way that $\mathbf{r}^{C_{0}}$ remains constant (or as long as $\mathbf{u}^{C_{0}} / O \approx \mathbf{0}$ at all times), then Eqs. (13) and (14) simplify further to

$\mathbf{r}^{C / O}=\frac{V_{\mathrm{o}}}{V} \mathbf{r}^{C_{\mathrm{o}}}-\frac{V_{B}}{V} \mathbf{r}^{B / O}$,

and

$\mathbf{u}^{C / O}=-\frac{V_{B}}{V} \mathbf{u}^{B / O}$.

Substituting Eqs. (15) and (16) into the full equation (Eq. 11) and setting $\mathbf{M}^{\mathrm{CV} / C}=\mathbf{0}$ (from Sect. 3.1), the moment on the body with respect to its center of mass can now be written as:

$$
\begin{aligned}
\mathbf{M}^{B}= & \frac{V_{B}}{V} \mathbf{u}^{B / O} \times \rho\left(\iint_{\mathrm{CS}} \mathbf{r}(\mathbf{u} \cdot \mathbf{n}) \mathrm{d} S+\iint_{\partial B} \mathbf{r}(\mathbf{u} \cdot \mathbf{n}) \mathrm{d} S\right) \\
& -\iint_{\mathrm{CS}}\left(\mathbf{r}-\frac{V_{\mathrm{o}}}{V} \mathbf{r}^{C_{\mathrm{o}}}+\frac{V_{B}}{V} \mathbf{r}^{B / O}\right) \times p \mathbf{n} \mathrm{d} S \\
& +\iint_{\mathrm{CS}}\left(\mathbf{r}-\frac{V_{\mathrm{o}}}{V} \mathbf{r}^{C_{\mathrm{o}}}+\frac{V_{B}}{V} \mathbf{r}^{B / O}\right) \times(\overline{\bar{\tau}} \cdot \mathbf{n}) \mathrm{d} S \\
& +\left(\frac{V_{\mathrm{o}}}{V} \mathbf{r}^{C_{\mathrm{o}}}-\frac{V_{B}}{V} \mathbf{r}^{B / O}\right) \times \rho \iint_{\mathrm{CS}} \mathbf{u}((\mathbf{u}-\mathbf{v}) \cdot \mathbf{n}) \mathrm{d} S \\
& +\left(\frac{V_{\mathrm{o}}}{V} \mathbf{r}^{C_{\mathrm{o}}}-\left(1+\frac{V_{B}}{V}\right) \mathbf{r}^{B / O}\right) \\
& \times \mathbf{F}+\left(\frac{V_{\mathrm{o}}}{V} \mathbf{r}^{C_{\mathrm{o}}}-\frac{V_{B}}{V} \mathbf{r}^{B / O}\right) \times \mathbf{g} \rho V .
\end{aligned}
$$

We are now ready to evaluate when the first term in Eq. (17), $\frac{V_{B}}{V} \mathbf{u}^{B / O} \times \rho\left(\iint_{\mathrm{CS}} \mathbf{r}(\mathbf{u} \cdot \mathbf{n}) \mathrm{d} S+\iint_{\partial B} \mathbf{r}(\mathbf{u} \cdot \mathbf{n}) \mathrm{d} S\right)$, can be neglected. We first apply Gauss's Theorem to reformulate the term as

$$
\begin{aligned}
& \frac{V_{B}}{V} \mathbf{u}^{B / O} \times \rho \iiint_{C V} \mathbf{u d} V \\
& =\frac{V_{B}}{V} \mathbf{u}^{B / O} \times \rho V \overline{\mathbf{u}}=\rho V_{B} \mathbf{u}^{B / O} \times \overline{\mathbf{u}},
\end{aligned}
$$

where $\overline{\mathbf{u}}=\frac{1}{V} \iiint_{C V} \mathbf{u d} V$ is the volume-averaged flow velocity in the CV. To evaluate when it is safe to neglect this term, we compare its magnitude to a reference moment-the product of a characteristic length scale of the body $l_{B}$ (such as the distance between the center of pressure of a force generating surface, such as a wing, from the body's center of mass) and the weight of the body. We now denote the density of the fluid as $\rho_{\mathrm{f}}$ and the density of the body as $\rho_{B}$ :

$\epsilon_{u}=\frac{\rho_{\mathrm{f}} V_{B} \mathbf{u}^{B / O} \times \overline{\mathbf{u}}}{l_{B} \rho_{B} V_{B} \mathbf{g}}=\frac{\rho_{\mathrm{f}} \mathbf{u}^{B / O} \times \overline{\mathbf{u}}}{l_{B} \rho_{B} \mathbf{g}}$. 
The first term in Eq. (17) can thus be neglected when the ratio $\epsilon_{u}=0$ or $\epsilon_{u} \ll 1$. For example, if the velocity of the body is zero (such as a model mounted on a sting), then $\mathbf{u}^{B / O}=0$ so $\epsilon_{u}=0$. Similarly, in still fluid experiments, as in aerodynamic force platform and many partical image velocimetry studies, $\overline{\mathbf{u}} \approx 0$ so $\epsilon_{u} \approx 0$, provided that the volume is large enough to not be dominated by the body's wake. The third case where $\epsilon_{u}$ will be small is when the body and fluid velocities are closely aligned $\left(\mathbf{u}^{B / O} \times \overline{\mathbf{u}} \approx 0\right)$, as is the case for forward motion in a wind tunnel or flow channel (again assuming that the volume is large enough that it contains more undisturbed freestream volume than disturbed/ wake flow volume). In general, if $\mathscr{O}\left(\mathbf{u}^{B / O} \times \overline{\mathbf{u}}\right) \leq \mathscr{O}\left(l_{B} \mathbf{g}\right)$, then $\mathscr{O}\left(\epsilon_{u}\right) \leq \mathscr{O}\left(\frac{\rho_{\mathrm{f}}}{\rho_{\mathrm{b}}}\right)$, so this term can also be neglected when the density of the fluid is much less than that of the body $\rho_{\mathrm{f}} \ll \rho_{\mathrm{b}}$. For instance, many animals have densities similar to water $\mathscr{O}\left(1000 \mathrm{~kg} \mathrm{~m}^{-3}\right)$, so when the fluid is air, $\frac{\rho_{\mathrm{f}}}{\rho_{\mathrm{b}}} \approx 0.001$

\subsection{Small body to control volume ratio}

It is generally possible in fluid experiments to define a $\mathrm{CV}$ with a much larger volume $V$ than the flow volume taken up by the body and its wake $V_{B}\left(\frac{V_{B}}{V} \ll 1\right)$. This means that $V=V_{\mathrm{o}}-V_{B} \approx V_{\mathrm{o}}$ and from Eq. (15), $\mathbf{r}^{C / O}=\frac{V_{\mathrm{o}}}{V} \mathbf{r}^{C_{\mathrm{o}}}-\frac{V_{B}}{V} \mathbf{r}^{B / O} \approx \mathbf{r}^{C_{\mathrm{o}}}$. If $\epsilon_{u} \approx 0$ based on the conditions described in the previous section, then $\frac{V_{B}}{V} \mathbf{u}^{B / O} \times \rho\left(\iint_{\mathrm{CS}} \mathbf{r}(\mathbf{u} \cdot \mathbf{n}) \mathrm{d} S+\iint_{\partial B} \mathbf{r}(\mathbf{u} \cdot \mathbf{n}) \mathrm{d} S\right) \approx 0$, and the expression for the moment on the body (Eq. 17) simplifies to:

$$
\begin{aligned}
\mathbf{M}^{B}= & -\iint_{\mathrm{CS}}\left(\mathbf{r}-\mathbf{r}^{C_{\mathrm{o}}}\right) \times p \mathbf{n} \mathrm{d} S \\
& +\iint_{\mathrm{CS}}\left(\mathbf{r}-\mathbf{r}^{C_{\mathrm{o}}}\right) \times(\overline{\bar{\tau}} \cdot \mathbf{n}) \mathrm{d} S \\
& +\mathbf{r}^{C_{\mathrm{o}}} \times \rho \iint_{\mathrm{CS}} \mathbf{u}((\mathbf{u}-\mathbf{v}) \cdot \mathbf{n}) \mathrm{d} S \\
& +\left(\mathbf{r}^{C_{\mathrm{o}}}-\mathbf{r}^{B / O}\right) \times \mathbf{F}+\mathbf{r}^{C_{\mathrm{o}}} \times \mathbf{g} \rho V .
\end{aligned}
$$

\subsection{Unsteady body moment}

In the second-to-last term in Eq. (11), the fluid force $\mathbf{F}$ includes an unsteady body force term (see Eq. 9), $\mathbf{U B F}=\rho \frac{\mathrm{d}}{\mathrm{d} t} \iint_{\partial B} \mathbf{r}(\mathbf{u} \cdot \mathbf{n}) \mathrm{d} S=\rho \frac{\mathrm{d}}{\mathrm{d} t} \iiint_{B} \mathbf{u d} V$, which describes the inertial force associated with the fluid displaced by the body volume $B$ (Lentink 2018). As detailed in Lentink (2018), the UBF can often be neglected, such as when the fluid-body density ratio is small. Here we add an additional case when it can be neglected in deriving $\mathbf{M}^{B}$. From Eq. (11), we see that the UBF does not contribute significantly to the moment on the body if $\left(\mathbf{r}^{C / O}-\mathbf{r}^{B / O}\right) \times \mathbf{U B F}=\mathbf{r}^{C / B} \times \mathbf{U B F}$ is small. To see when this is the case, we can first write the moment resulting from the unsteady body force as:

$\mathbf{r}^{C / B} \times \mathbf{U B F}=\mathbf{r}^{C / B} \times \rho \frac{\mathrm{d}}{\mathrm{d} t} \iiint_{B} \mathbf{u d} V=\mathbf{r}^{C / B} \times \rho V_{B} \overline{\mathbf{a}}_{B}$,

where $\overline{\mathbf{a}}_{B}=\frac{1}{V} \frac{\mathrm{d}}{\mathrm{d} t} \iiint_{B} u \mathrm{~d} V$ is the volume-averaged acceleration of the displaced fluid. We can then compare this term with the reference moment first introduced in Sect. 3.2,

$\epsilon_{\mathbf{U B F}}=\frac{\mathbf{r}^{C / B} \times \rho_{\mathrm{f}} V_{B} \overline{\mathbf{a}}_{B}}{l_{B} \rho_{B} V_{B} \mathbf{g}}=\frac{\mathbf{r}^{C / B} \times \rho_{\mathrm{f}} \overline{\mathbf{a}}_{B}}{l_{B} \rho_{B} \mathbf{g}}$.

Thus if the average body acceleration is aligned with a line connecting the body's center of mass and the control volume's center of mass (i.e. $\mathbf{r}^{C / B} \times \overline{\mathbf{a}}_{B}=0$ ), then the $\mathbf{U B F}$ can be neglected without loss of accuracy. The fluid force in Eq. (11) can then be simplified to:

$$
\begin{aligned}
\mathbf{F}= & -\iint_{\mathrm{CS}} p \mathbf{n d} S+\iint_{\mathrm{CS}}(\overline{\bar{\tau}} \cdot \mathbf{n}) \mathrm{d} S \\
& -\rho \iint_{\mathrm{CS}} \mathbf{u}((\mathbf{u}-\mathbf{v}) \cdot \mathbf{n})-\rho \frac{\mathrm{d}}{\mathrm{d} t} \iint_{\mathrm{CS}} \mathbf{r}(\mathbf{u} \cdot \mathbf{n}) \mathrm{d} S .
\end{aligned}
$$

\subsection{Stationary outer control surface}

For experiments in which the outer boundary is formed by stationary solid walls, as is the case for the aerodynamic force platform, $\mathbf{u}=\mathbf{0}$ at the outer control surface CS (Fig. 1d). Equation (18) then becomes:

$$
\begin{aligned}
\mathbf{M}^{B}= & -\iint_{\mathrm{CS}}\left(\mathbf{r}-\mathbf{r}^{C_{\mathrm{o}}}\right) \times p \mathbf{n} \mathrm{d} S \\
& +\iint_{\mathrm{CS}}\left(\mathbf{r}-\mathbf{r}^{C_{\mathrm{o}}}\right) \times(\overline{\bar{\tau}} \cdot \mathbf{n}) \mathrm{d} S \\
& +\left(\mathbf{r}^{C_{\mathrm{o}}}-\mathbf{r}^{B / O}\right) \times \mathbf{F}+\mathbf{r}^{C_{\mathrm{o}}} \times \mathbf{g} \rho V .
\end{aligned}
$$

The final gravity term represents the net force on the control volume due to its submersion in fluid (e.g. air or water) that is partially displaced by the body inside. If measurements are initially tared while the body and the fluid are at rest, this gravity term, which is the only time-independent term, will drop out. Then, writing $\mathbf{r}-\mathbf{r}^{C_{\mathrm{o}}}$ more concisely as $\mathbf{r}^{P / C_{\mathrm{o}}}$, and $\mathbf{r}^{C_{\mathrm{o}}}-\mathbf{r}^{B / O}$ as $\mathbf{r}^{C_{\mathrm{o}} / B}$, the moment on the body about its center of mass becomes:

$$
\begin{aligned}
\mathbf{M}^{B}= & -\iint_{\mathrm{CS}} \mathbf{r}^{P / C_{\mathrm{o}}} \times p \mathbf{n d} S \\
& +\iint_{\mathrm{CS}} \mathbf{r}^{P / C_{\mathrm{o}}} \times(\overline{\bar{\tau}} \cdot \mathbf{n}) \mathrm{d} S+\mathbf{r}^{C_{\mathrm{o}} / B} \times \mathbf{F}
\end{aligned}
$$

where the fluid force on the body $\mathbf{F}$ (from Eq. 9) similarly simplifies to $\mathbf{F}=-\iint_{\mathrm{CS}} p \mathbf{n d} S+\iint_{\mathrm{CS}}(\overline{\bar{\tau}} \cdot \mathbf{n}) \mathrm{d} S-\mathbf{U B F}$, or if 
the unsteady body force can be neglected (as discussed in the previous section),

$\mathbf{F}=-\iint_{\mathrm{CS}} p \mathbf{n} \mathrm{d} S+\iint_{\mathrm{CS}}(\overline{\bar{\tau}} \cdot \mathbf{n}) \mathrm{d} S$.

Together, Eqs. (20) and (21) are the most simplified and yet still widely applicable versions of the full fluid moment and fluid force equations (Eqs. 9 and 11). We next consider the implications of two less commonly encountered conditions - the presence of a liquid-gas interface, and the case of a variable body volume.

\subsection{Liquid-gas interface}

The equations and analysis in the previous sections also hold for bodies traveling at a liquid-gas interface, where most of the body is surrounded by gas and only a small segment by liquid. Following Lentink (2018), we consider the control surface $\partial V$ in terms of the free surface (with liquid on one side and gas on the other) $F S$, the body surface in contact forces dominate (Bush and Hu 2006; Lentink 2018). At small Weber and Bond numbers, surface tension becomes important and necessitates the calculation of surface curvature. However, the contribution of surface tension to $\mathbf{M}^{B}$ will remain small if the force is oriented mostly towards the body's center of mass.

The center of mass of the control volume will be determined primarily by the liquid volume, so only the submerged volume of the body $V_{\mathrm{s}}$ will have appreciable effects on $\mathbf{r}^{C / O}$ and $\mathbf{u}^{C / O}$. This means that the requirement that $\frac{V_{B}}{V} \ll 1$ used to simplify the moment equation in Sect. 3.3 can be modified to $\frac{V_{\mathrm{s}}}{V} \ll 1$, so the simplified formulation can still hold for larger bodies (or smaller control volumes) as long as the submerged portion of the body is small in comparison.

\subsection{Variable body volume}

In the majority of this section, we assumed that body volume remains constant, but we now consider the case where the body volume is not constant. Taking the time derivative of $\mathbf{r}^{C / O}$ from Eq. (13), the velocity of the fluid center of mass becomes:

$$
\begin{aligned}
\mathbf{u}^{C / O} & =\frac{\mathrm{d}}{\mathrm{d} t}\left(\frac{\mathbf{r}^{C_{\mathrm{o}}} V_{\mathrm{o}}-\mathbf{r}^{B / O} V_{B}}{V_{\mathrm{o}}-V_{B}}\right) \\
& =\frac{\left(V_{\mathrm{o}}-V_{B}\right)\left(-\mathbf{r}^{B / O} \dot{V}_{B}-\mathbf{u}^{B / O} V_{B}\right)-\left(\mathbf{r}^{C_{\mathrm{o}}} V_{\mathrm{o}}-\mathbf{r}^{B / O} V_{B}\right)\left(-\dot{V}_{B}\right)}{\left(V_{\mathrm{o}}-V_{B}\right)^{2}} \\
& =\frac{-\mathbf{r}^{B / O} V_{\mathrm{o}} \dot{V}_{B}-\mathbf{u}^{B / O} V_{\mathrm{o}} V_{B}+\mathbf{r}^{B / O} V_{B} \dot{V}_{B}+\mathbf{u}^{B / O} V_{B}^{2}+\mathbf{r}^{C_{\mathrm{o}}} V_{\mathrm{o}} \dot{V}_{B}-\mathbf{r}^{B / O} V_{B} \dot{V}_{B}}{\left(V_{\mathrm{o}}-V_{B}\right)^{2}} \\
& =\frac{-\mathbf{r}^{B / O} V_{\mathrm{o}} \dot{V}_{B}-\mathbf{u}^{B / O} V_{\mathrm{o}} V_{B}+\mathbf{u}^{B / O} V_{B}^{2}+\mathbf{r}^{C_{\mathrm{o}}} V_{\mathrm{o}} \dot{V}_{B}}{\left(V_{\mathrm{o}}-V_{B}\right)^{2}} \\
& =\frac{-\mathbf{u}^{B / O} V_{B}\left(V_{\mathrm{o}}-V_{B}\right)+V_{\mathrm{o}} \dot{V}_{B}\left(\mathbf{r}^{C_{\mathrm{o}}}-\mathbf{r}^{B / O}\right)}{\left(V_{\mathrm{o}}-V_{B}\right)^{2}} \\
& =-\frac{V_{B}}{\left(V_{\mathrm{o}}-V_{B}\right)} \mathbf{u}^{B / O}+\frac{V_{\mathrm{o}} \dot{V}_{B}}{\left(V_{\mathrm{o}}-V_{B}\right)^{2}} \mathbf{r}^{C_{\mathrm{o}} / B},
\end{aligned}
$$

with the water $\partial B_{w}$, the body surface in contact with the air $\partial B_{a}$, and the remaining outer control surface that encloses the control volume CS. However, the control surface integrals corresponding to $F S$ and $\partial B_{a}$ do not contribute significantly to the net moment acting on the body. As described in Lentink (2018), stresses on the gas side of the interface do not contribute to the net force or moment on the body, because stress in the gas is constant and equal to $-p_{0} \overline{\overline{\mathbf{I}}}$ everywhere around the control surface (where $\overline{\overline{\mathbf{I}}}$ is the identity tensor), and shear stress is negligible. For the moment analysis, this gives: $-\iint_{\mathrm{FS}} \mathbf{r} \times(p \cdot \mathbf{n}) \mathrm{d} S+\iint_{\mathrm{FS}} \mathbf{r} \times(\overline{\overline{\boldsymbol{\tau}}} \cdot \mathbf{n}) \mathrm{d} S=0$. There is no flow through the free surface, so $-\rho \iint_{\mathrm{FS}} \mathbf{r} \times[(\mathbf{u}-\mathbf{v}) \cdot \mathbf{n}] \mathrm{d} S=0$.

While there is surface tension acting on the body at the liquid-gas interface between $\partial B_{w}$ and $\partial B_{a}$, it is negligible at large Weber and Bond numbers, wherein fluid inertia and buoyancy or using $V=V_{\mathrm{o}}-V_{B}$,

$\mathbf{u}^{C / O}=-\frac{V_{B}}{V} \mathbf{u}^{B / O}+\frac{V_{\mathrm{o}} \dot{V}_{B}}{V^{2}} \mathbf{r}^{C_{\mathrm{o}} / B}$.

From Eq. (11), the moment contribution related to $\mathbf{u}^{C / O}$ depends on its cross product with $\rho \iiint_{C V} \mathbf{u d V}$, so the moment contribution from changes in the body volume $\dot{V}_{B}$ will equal:

$$
\begin{aligned}
& \frac{V_{\mathrm{o}} \dot{V}_{B}}{V^{2}} \mathbf{r}^{C_{\mathrm{o}} / B} \times \rho \iiint_{C V} \mathbf{u d} V=\frac{V_{\mathrm{o}} \dot{V}_{B}}{V^{2}} \mathbf{r}^{C_{\mathrm{o}} / B} \times \rho V \overline{\mathbf{u}} \\
& \quad=\rho \frac{V_{\mathrm{o}} \dot{V}_{B}}{V} \mathbf{r}^{C_{\mathrm{o}} / B} \times \overline{\mathbf{u}} .
\end{aligned}
$$

Comparing to the reference moment $l_{B} \rho_{B} V_{B} \mathbf{g}$, 
$\epsilon_{\dot{V}_{B}}=\frac{\rho_{\mathrm{f}} V_{\mathrm{o}} \dot{V}_{B} \mathbf{r}^{C_{\mathrm{o}} / B} \times \overline{\mathbf{u}}}{V l_{B} \rho_{B} V_{B} \mathbf{g}}=\frac{V_{\mathrm{o}}}{V} \frac{\rho_{\mathrm{f}}}{\rho_{B}} \frac{\dot{V}_{B}}{V_{B}} \frac{\mathbf{r}^{C_{\mathrm{o}} / B} \times \overline{\mathbf{u}}}{l_{B} \mathbf{g}}$.

The relative error from neglecting changes in body volume, $\epsilon_{\dot{V}_{B}}$, thus depends on the product of four ratios. Since $V_{\mathrm{o}}=V+V_{B}$, the first ratio $\frac{V_{\mathrm{o}}}{V}>1$, but as long as $V_{B}$ is small $\left(\frac{V_{B}}{V} \leq \mathscr{O}(1)\right)$, then $\frac{V_{0}}{V} \sim \mathscr{O}(1)$. Thus if any one of the remaining three ratios is near zero $(\ll 1)$ while the product of the other two are $\leq \mathscr{O}(1)$, then body volume changes can be neglected. For instance, if $\mathscr{O}\left(\frac{V_{\mathrm{o}}}{V} \frac{\dot{V}_{B}}{V_{B}} \frac{\mathbf{r}_{\mathrm{O}} / B}{l_{B} \mathbf{g}}\right) \leq 1$, then $\mathscr{O}\left(\epsilon_{\dot{V}_{B}}\right) \leq \mathscr{O}\left(\frac{\rho_{\mathrm{f}}}{\rho_{B}}\right)$, so variable body volume can often be neglected when the fluid-body density ratio is small, as is commonly the case when the fluid is air (as discussed in Sec. 3.2). Similarly, $\epsilon_{\dot{V}_{B}}$ will generally be small if the time rate of change of the body volume is small relative to the body volume itself, $\mathscr{O}\left(\frac{\dot{V}_{B}}{V_{B}}\right) \ll 1$, or if the volume-averaged fluid velocity is zero or closely aligned with $\mathbf{r}^{C_{0} / B}$, $\mathscr{O}\left(\mathbf{r}^{C_{\mathrm{o}}} / B \times \overline{\mathbf{u}}\right) \ll 1$. If, however, $\frac{V_{\mathrm{o}} \dot{V}_{B}}{V^{2}} \mathbf{r}^{C_{\mathrm{o}}} / B \times \rho \iiint_{C V} \mathbf{u d} V$ is large, then body volume changes will need to be quantified.

\subsection{Summary table of simplifying conditions for fluid moment and force formulations}

In the following table, we summarize the various conditions described in this section and their implications on the full fluid moment (Eq. 11) and fluid force (Eq. 9) formulations, which we repeat here for reference:

$$
\begin{aligned}
& \mathbf{M}^{B}=-\underbrace{\mathbf{M}^{\mathrm{CV} / C}}_{(A)}-\underbrace{\mathbf{u}^{C / O} \times \rho\left(\iint_{\mathrm{CS}} \mathbf{r}(\mathbf{u} \cdot \mathbf{n}) \mathrm{d} S+\iint_{\partial B} \mathbf{r}(\mathbf{u} \cdot \mathbf{n}) \mathrm{d} S\right)}_{(\mathrm{B})} \\
& -\underbrace{\iint_{\mathrm{CS}}\left(\mathbf{r}-\mathbf{r}^{C / O}\right) \times p \mathbf{n d} S+\iint_{\mathrm{CS}}\left(\mathbf{r}-\mathbf{r}^{C / O}\right) \times(\overline{\bar{\tau}} \cdot \mathbf{n}) \mathrm{d} S}_{\text {(C) }} \\
& +\underbrace{\mathbf{r}^{C / O} \times \rho \iint_{\mathrm{CS}} \mathbf{u}((\mathbf{u}-\mathbf{v}) \cdot \mathbf{n}) \mathrm{d} S}_{(\mathrm{D})} \\
& +\underbrace{\left(\mathbf{r}^{C / O}-\mathbf{r}^{B / O}\right) \times \mathbf{F}}_{(\mathrm{E})}+\underbrace{\mathbf{r}^{C / O} \times \mathbf{g} \rho V}_{(\mathrm{F})} . \\
& \mathbf{F}=\underbrace{-\iint_{\mathrm{CS}} p \mathbf{n} \mathrm{d} S+\iint_{\mathrm{CS}}(\overline{\bar{\tau}} \cdot \mathbf{n}) \mathrm{d} S}_{(\mathrm{G})}-\underbrace{\rho \frac{\mathrm{d}}{\mathrm{d} t} \iint_{\partial B} \mathbf{r}(\mathbf{u} \cdot \mathbf{n}) \mathrm{d} S}_{(\mathrm{H})} \\
& -\underbrace{\rho \iint_{\mathrm{CS}} \mathbf{u}((\mathbf{u}-\mathbf{v}) \cdot \mathbf{n})-\rho \frac{\mathrm{d}}{\mathrm{d} t} \iint_{\mathrm{CS}} \mathbf{r}(\mathbf{u} \cdot \mathbf{n}) \mathrm{d} S}_{(\mathrm{I})} .
\end{aligned}
$$

\section{Aerodynamic force platform formulations}

We now show more explicitly how the instantaneous net forces and moments $\mathbf{M}^{B}$ on a moving, deformable body can be directly calculated using measurements from an aerodynamic force platform (AFP). We then show an example of the resulting fluid moment generated by a bird's wingbeat in the center of an AFP.

The AFP uses solid instrumented plates to mechanically integrate pressure and shear acting on the top, bottom, front, rear, and side walls of a control volume (Fig. 2). Taking into account these stationary walls that form the outer control surface CS and taring the gravity term out when the body and fluid are both at rest, we can start from Eq. (20):

$$
\begin{aligned}
\mathbf{M}^{B}(t)= & -\iint_{\mathrm{CS}} \mathbf{r}^{P / C_{\mathrm{o}}}(t) \times p(t) \mathbf{n} \mathrm{d} S \\
& +\iint_{\mathrm{CS}} \mathbf{r}^{P / C_{\mathrm{o}}}(t) \times(\overline{\bar{\tau}}(t) \cdot \mathbf{n}) \mathrm{d} S+\mathbf{r}^{C_{\mathrm{o}} / B}(t) \times \mathbf{F}(t),
\end{aligned}
$$

where again $\mathbf{r}^{P / C_{0}}$ is the position of a surface element $\mathrm{d} S$ from the position of the CV's center of mass without the body present $C_{\mathrm{o}}$, and $\mathbf{r}^{C_{\mathrm{o}} / B}$ is the position of $C_{\mathrm{o}}$ relative to the center of mass of the body $B$. For all flying bodies heavier than air, the fluid force is given by Eq. 21 (Lentink 2018):

$\mathbf{F}(t)=-\iint_{\mathrm{CS}} p(t) \mathbf{n} \mathrm{d} S+\iint_{\mathrm{CS}}(\overline{\bar{\tau}}(t) \cdot \mathbf{n}) \mathrm{d} S$,

so the formulation for the moment about the body becomes (Fig. 1c):

$$
\begin{aligned}
\mathbf{M}^{B}(t)= & -\iint_{\mathrm{CS}} \mathbf{r}^{P / C_{\mathrm{o}}} \times p \mathbf{n} \mathrm{d} S \\
& +\iint_{\mathrm{CS}} \mathbf{r}^{P / C_{\mathrm{o}}} \times(\overline{\bar{\tau}} \cdot \mathbf{n}) \mathrm{d} S+\mathbf{r}^{C_{\mathrm{o}} / B} \\
& \times\left(-\iint_{\mathrm{CS}} p \mathbf{n} \mathrm{d} S+\iint_{\mathrm{CS}}(\overline{\bar{\tau}} \cdot \mathbf{n}) \mathrm{d} S\right) \\
= & -\iint_{\mathrm{CS}} \mathbf{r}^{P / C_{\mathrm{o}}} \times p \mathbf{n} \mathrm{d} S \\
& +\iint_{\mathrm{CS}} \mathbf{r}^{P / C_{\mathrm{o}}} \times(\overline{\bar{\tau}} \cdot \mathbf{n}) \mathrm{d} S \\
& -\iint_{\mathrm{CS}} \mathbf{r}^{C_{\mathrm{o}} / B} \times p \mathbf{n} \mathrm{d} S \\
& +\iint_{\mathrm{CS}} \mathbf{r}^{C_{\mathrm{o}} / B} \times(\overline{\bar{\tau}} \cdot \mathbf{n}) \mathrm{d} S \\
= & -\iint_{\mathrm{CS}}\left(\mathbf{r}^{P / C_{\mathrm{o}}}+\mathbf{r}^{C_{\mathrm{o}} / B}\right) \times p \mathbf{n} \mathrm{d} S \\
& +\iint_{\mathrm{CS}}\left(\mathbf{r}^{P / C_{\mathrm{o}}}+\mathbf{r}^{C_{\mathrm{o}} / B}\right) \times(\overline{\bar{\tau}} \cdot \mathbf{n}) \mathrm{d} S \\
= & -\iint_{\mathrm{CS}} \mathbf{r}^{P / B}(t) \times p(t) \mathbf{n} \mathrm{d} S \\
& +\iint_{\mathrm{CS}} \mathbf{r}^{P / B}(t) \times(\overline{\bar{\tau}}(t) \cdot \mathbf{n}) \mathrm{d} S .
\end{aligned}
$$


We expect that any error from using this simplified formulation in place of the full equations (Eqs. 9 and 11) should be less than $1 \%$, because the neglected terms are either effectively zero (in the theoretical limit) or are on the same order as the small fluid-body density ratio $\left(\frac{\rho_{\mathrm{f}}}{\rho_{\mathrm{b}}} \approx 0.001\right)$.

Before we can evaluate these integrals using measurements from the AFP, we must first expand them for each surface of the AFP, e.g. the top plate (TP), bottom plate (BP), front plate (FP), rear plate (RP), left wall (LW), and right wall (RW):

$\mathbf{M}^{B}(t)$

$$
\begin{aligned}
= & -\iint_{\mathrm{CS}} \mathbf{r}^{P / B} \times p \mathbf{n} \mathrm{d} S+\iint_{\mathrm{CS}} \mathbf{r}^{P / B} \times(\overline{\bar{\tau}} \cdot \mathbf{n}) \mathrm{d} S \\
= & -\iint_{\mathrm{TP}} \mathbf{r}^{P / B} \times p \mathbf{n} \mathrm{d} S+\iint_{\mathrm{TP}} \mathbf{r}^{P / B} \times(\overline{\bar{\tau}} \cdot \mathbf{n}) \mathrm{d} S \\
& -\iint_{\mathrm{BP}} \mathbf{r}^{P / B} \times p \mathbf{n} \mathrm{d} S+\iint_{\mathrm{BP}} \mathbf{r}^{P / B} \times(\overline{\bar{\tau}} \cdot \mathbf{n}) \mathrm{d} S \\
& -\iint_{\mathrm{FP}} \mathbf{r}^{P / B} \times p \mathbf{n} \mathrm{d} S+\iint_{\mathrm{FP}} \mathbf{r}^{P / B} \times(\overline{\bar{\tau}} \cdot \mathbf{n}) \mathrm{d} S \\
& -\iint_{\mathrm{RP}} \mathbf{r}^{P / B} \times p \mathbf{n} \mathrm{d} S+\iint_{\mathrm{RP}} \mathbf{r}^{P / B} \times(\overline{\bar{\tau}} \cdot \mathbf{n}) \mathrm{d} S \\
& -\iint_{\mathrm{LW}} \mathbf{r}^{P / B} \times p \mathbf{n} \mathrm{d} S+\iint_{\mathrm{LW}} \mathbf{r}^{P / B} \times(\overline{\bar{\tau}} \cdot \mathbf{n}) \mathrm{d} S \\
& -\iint_{\mathrm{RW}} \mathbf{r}^{P / B} \times p \mathbf{n} \mathrm{d} S+\iint_{\mathrm{RW}} \mathbf{r}^{P / B} \times(\overline{\bar{\tau}} \cdot \mathbf{n}) \mathrm{d} S .
\end{aligned}
$$

If we express the moment contribution of each plate to the total moment about $B$ as $\mathbf{M}^{\text {Plate } / B}(t)=-\iint_{\text {Plate }} \mathbf{r}^{P / B} \times p \mathbf{n d} S+\iint_{\text {Plate }} \mathbf{r}^{P / B} \times(\overline{\bar{\tau}} \cdot \mathbf{n}) \mathrm{d} S$, then we are left with:

$$
\begin{aligned}
\mathbf{M}^{B}(t)= & \mathbf{M}^{\mathrm{TP} / B}(t)+\mathbf{M}^{\mathrm{BP} / B}(t)+\mathbf{M}^{\mathrm{FP} / B}(t) \\
& +\mathbf{M}^{\mathrm{RP} / B}(t)+\mathbf{M}^{\mathrm{LW} / B}(t)+\mathbf{M}^{\mathrm{RW} / B}(t) .
\end{aligned}
$$

To calculate the moment contribution of each plate in terms of the measured forces on the plate, we apply the shift theorem (Mitiguy 2015) to write:

$\mathbf{M}^{\text {Plate } / B}=\mathbf{M}^{\text {Plate } / Q}+\mathbf{r}^{Q / B} \times \mathbf{F}^{\text {Plate }}$,

where $\mathbf{M}^{\text {Plate } / Q}$ is the moment of the plate about a point $Q$ (which we will choose to be the zero moment point on the plate, as detailed below), $\mathbf{r}^{Q / B}$ is the position vector from $B$ to $Q$, and $\mathbf{F}^{\text {Plate }}$ is the net fluid force acting on the plate, $\mathbf{F}^{\text {Plate }}=-\iint_{\text {Plate }} p \mathbf{n d} S+\iint_{\text {Plate }}(\overline{\bar{\tau}} \cdot \mathbf{n}) \mathrm{d} S$. The AFP force plates are each instrumented by three force/torque sensors, which enables us to recover the net force $\mathbf{F}^{\text {Plate }}$ and where the center of force acts on each plate (i.e. the position of the zero moment point $Q$ ). By defining $Q$ as the zeromoment point, $\mathbf{M}^{\text {Plate } / Q}=0$, we have all the needed information to find the moment contribution from each plate, $\mathbf{M}^{\text {Plate } / B}=\mathbf{r}^{Q / B} \times \mathbf{F}^{\text {Plate }}=\left(\mathbf{r}^{Q / O}-\mathbf{r}^{B / O}\right) \times \mathbf{F}^{\text {Plate }}$.
We denote the position vectors from the origin of the AFP to these zero-moment points $\left(\mathbf{r}^{Q^{\text {Plate }} / O}\right)$ on the top, bottom, front, rear, left, and right plates as $\mathbf{r}^{\mathrm{TP}}, \mathbf{r}^{\mathrm{BP}}, \mathbf{r}^{\mathrm{FP}}$, $\mathbf{r}^{\mathrm{RP}}, \mathbf{r}^{\mathrm{LW}}, \mathbf{r}^{\mathrm{RW}}$ (respectively), and the net fluid force on each plate as $\mathbf{F}^{\mathrm{TP}}, \mathbf{F}^{\mathrm{BP}}, \mathbf{F}^{\mathrm{FP}}, \mathbf{F}^{\mathrm{RP}}, \mathbf{F}^{\mathrm{LW}}, \mathbf{F}^{\mathrm{RW}}$ (Fig. 2) to write Eq. (23) as:

$$
\begin{aligned}
\mathbf{M}^{B}(t)= & \left(\mathbf{r}^{\mathrm{TP}}-\mathbf{r}^{B / O}\right) \times \mathbf{F}^{\mathrm{TP}}+\left(\mathbf{r}^{\mathrm{BP}}-\mathbf{r}^{B / O}\right) \times \mathbf{F}^{\mathrm{BP}} \\
& +\left(\mathbf{r}^{\mathrm{FP}}-\mathbf{r}^{B / O}\right) \times \mathbf{F}^{\mathrm{FP}}+\left(\mathbf{r}^{\mathrm{RP}}-\mathbf{r}^{B / O}\right) \times \mathbf{F}^{\mathrm{RP}} \\
& +\left(\mathbf{r}^{\mathrm{LW}}-\mathbf{r}^{B / O}\right) \times \mathbf{F}^{\mathrm{LW}}+\left(\mathbf{r}^{\mathrm{RW}}-\mathbf{r}^{B / O}\right) \times \mathbf{F}^{\mathrm{RW}}
\end{aligned}
$$

We next show how to derive $\mathbf{r}^{\mathrm{TP}}, \mathbf{r}^{\mathrm{BP}}, \mathbf{r}^{\mathrm{FP}}, \mathbf{r}^{\mathrm{RP}}, \mathbf{r}^{\mathrm{LW}}$, $\mathbf{r}^{\mathrm{RW}}$ based on the forces $\mathbf{F}_{1}, \mathbf{F}_{2}, \mathbf{F}_{3}$ and torques $\mathbf{T}_{1}, \mathbf{T}_{2}, \mathbf{T}_{3}$ measured by the three sensors that support each plate. We start with a force and moment balance for a single plate:

$$
\begin{aligned}
& \Sigma \mathbf{F}= \mathbf{F}^{\text {Plate }}+\mathbf{F}_{1}+\mathbf{F}_{2}+\mathbf{F}_{3}=0 \\
& \mathbf{F}^{\text {Plate }}=-\left(\mathbf{F}_{1}+\mathbf{F}_{2}+\mathbf{F}_{3}\right) . \\
& \Sigma \mathbf{M}^{\text {Plate } / Q}=\left(\mathbf{r}_{1}-\mathbf{r}^{Q / O}\right) \times \mathbf{F}_{1} \\
&+\left(\mathbf{r}_{2}-\mathbf{r}^{Q / O}\right) \times \mathbf{F}_{2} \\
&+\left(\mathbf{r}_{3}-\mathbf{r}^{Q / O}\right) \times \mathbf{F}_{3} \\
&+\mathbf{T}_{1}+\mathbf{T}_{2}+\mathbf{T}_{3}=0 \\
& \mathbf{r}_{1} \times \mathbf{F}_{1}+\mathbf{r}_{2} \times \mathbf{F}_{2}+\mathbf{r}_{3} \times \mathbf{F}_{3} \\
&+\mathbf{T}_{1}+\mathbf{T}_{2}+\mathbf{T}_{3} \\
&=\mathbf{r}^{Q / O} \times \underbrace{\left(\mathbf{F}_{1}+\mathbf{F}_{2}+\mathbf{F}_{3}\right.}_{-\mathbf{F}^{\text {Plate }}}),
\end{aligned}
$$

where $\mathbf{r}_{1}, \mathbf{r}_{2}, \mathbf{r}_{3}$ are the sensor positions relative to the AFP origin. Separating out the $x, y, z$ components, we can express Eq. (25) as three separate scalar equations, with $\mathbf{r}^{Q / O}=<x_{Q}, y_{Q}, z_{Q}>$, the components of the net force on the plate $\mathbf{F}^{\text {Plate }}=\left\langle F_{x}^{P}, F_{y}^{P}, F_{z}^{P}\right\rangle$, and the sensor positions $\mathbf{r}_{s}=\left\langle x_{s}, y_{s}, z_{s}\right\rangle$, forces $\mathbf{F}_{s}=\left\langle F_{x, s}, F_{y, s}, F_{z, s}\right\rangle$, and torques $\mathbf{T}_{s}=\left\langle T_{x, s}, T_{y, s}, T_{z, s}\right\rangle$ with $\mathrm{s}=1,2,3$ :

x-component: $\Sigma_{s=1}^{3}\left(y_{s} F_{z, s}-z_{s} F_{y, s}+T_{x, s}\right)=-y_{Q} F_{z}^{P}+z_{Q} F_{y}^{P}$

$y$-component: $\Sigma_{s=1}^{3}\left(z_{s} F_{x, s}-x_{s} F_{z, s}+T_{y, s}\right)=-z_{Q} F_{x}^{P}+x_{Q} F_{z}^{P}$

$z$-component: $\Sigma_{s=1}^{3}\left(x_{s} F_{y, s}-y_{s} F_{x, s}+T_{z, s}\right)=-x_{Q} F_{y}^{P}+y_{Q} F_{x}^{P}$,

where $F_{x}^{P}=-\left(F_{x, 1}+F_{x, 2}+F_{x, 3}\right), F_{y}^{P}=-\left(F_{y, 1}+F_{y, 2}+F_{y, 3}\right)$, and $F_{z}^{P}=-\left(F_{z, 1}+F_{z, 2}+F_{z, 3}\right)$. These equations can be used to derive the zero-moment points on any instrumented surface of the AFP. Since the forces and torques are measured, we then have three equations for each plate to solve for the 
coordinates of $Q^{\text {Plate }}\left(x_{Q}, y_{Q}, z_{Q}\right)$. We note that because the fluid force must act against the surface of the plate, one of these coordinates is already known for each plate based on the design of the AFP $\left(z_{Q}\right.$ is fixed for the top plate and bottom plate and has known dimensions in meters, and similarly $x_{Q}$ is known for the rear plate and front plate, and $y_{Q}$ is known for the side plates). As a result, only two of the equations are actually necessary to solve for $\mathbf{r}^{Q}$ for each plate. For example, solving these equations for the zero-moment point on the top plate $\mathbf{r}^{\mathrm{TP}}$ (Fig. 2), we get from Eq. (26),

$y_{Q}=\frac{\Sigma_{s=1}^{3}\left(y_{s} F_{z, s}-z_{s} F_{y, s}+T_{x, s}\right)-z_{Q} F_{y}^{P}}{-F_{z}^{P}}$,

and from Eq. (27),

$x_{Q}=\frac{\Sigma_{s=1}^{3}\left(z_{s} F_{x, s}-x_{s} F_{z, s}+T_{y, s}\right)+z_{Q} F_{x}^{P}}{F_{z}^{P}}$.

We can similarly calculate these position vectors for the other five plates, $\mathbf{r}^{\mathrm{TP}}, \mathbf{r}^{\mathrm{FP}}, \mathbf{r}^{\mathrm{RP}}, \mathbf{r}^{\mathrm{LW}}$, and $\mathbf{r}^{\mathrm{RW}}$. Equation (22) can then be used to calculate the net fluid moment on a body about its center of mass. Therefore, by measuring the forces that act on the AFP plates, we are able to calculate the net instantaneous force and moment that a deforming body generates as it moves freely inside of the AFP. These equations also apply to applications in other fluids such as water.

Finally, we demonstrate the result of applying these formulations for the wingbeat of a Pacific parrotlet flying near the center of a 2D aerodynamic force platform (Fig. 3). We have previously shown experimental results for fluid forces measured by 1D aerodynamic force platforms that measure vertical forces (Lentink et al. 2015; Chin and Lentink 2017; Ingersoll and Lentink 2018; Ingersoll et al. 2018). The 2D AFP includes instrumented top, bottom, front, and rear plates for measuring both net vertical and horizontal forces (additional details on this setup will be published elsewhere). We trained five Pacific parrotlets (Forpus coelestis; $30.7 \pm 2.6 \mathrm{~g}, 20 \mathrm{~Hz}$ wingbeat frequency, $22.0 \pm 1.5 \mathrm{~cm}$ mid-downstroke wingspan) to fly between two perches in the AFP using habituation and positive reinforcement (food and water provided ad libitum; cages have enrichment, animals

Table 1 Summary of simplifications that can be made to the full formulations for fluid force and fluid moment acting on a body submerged in fluid or between two fluid interfaces

\begin{tabular}{|c|c|c|c|}
\hline Sec. & Conditions & Implications & Typical applications \\
\hline 3.1 & Non-rotating control volume $\left(\omega^{\mathrm{CV}}=0, \boldsymbol{\alpha}^{\mathrm{CV}}=0\right)$ & $\operatorname{Term}(\mathrm{A})=0$ & ve, an, ob PIV, AFP \\
\hline 3.2 & $\begin{array}{l}\text { Constant control volume } V \text { and constant body volume } \\
V_{B} \text { with: body velocity } \approx 0 \text {, or fluid velocity } \approx 0 \text {, } \\
\text { or the body and fluid velocities are closely aligned, } \\
\text { or the average body density is much larger than the } \\
\qquad\left(\epsilon_{u}=\frac{\rho_{\mathrm{f}} \mathbf{u}^{B / O} \times \overline{\mathbf{u}}}{\rho_{B} l_{B} \mathbf{g}} \ll 1\right)\end{array}$ & $\operatorname{Term}(B)=0$ & ve, an, ob PIV, AFP \\
\hline 3.3 & Small body to control volume ratio $\left(V_{B} / V \ll 1\right)$ & $\begin{array}{l}\text { If conditions } 3.1-3.3 \text { are true, then Terms }(\mathrm{A}) \text { and }(\mathrm{B})=0 \text {, and the position of the } \\
\text { CV's center of mass } \mathbf{r}^{C / O} \text { can be approximated by } \mathbf{r}^{C_{0}} \text {, its position when no body } \\
\text { is present (Eq. } 11 \text { simplifies to Eq. } 18 \text { ) }\end{array}$ & ve, an, ob PIV, AFP \\
\hline 3.4 & $\begin{array}{l}\text { The unsteady body force (the force associated with } \\
\text { the acceleration of the fluid volume displaced by } \\
\text { the body) is negligible, as is the case when the aver- } \\
\text { age body density is much larger than the average } \\
\text { fluid density. Alternatively, the body accelerates } \\
\text { towards or away from the CV's center of mass. } \\
\left(\epsilon_{\mathbf{U B F}}=\frac{\rho_{\mathrm{f}} \mathbf{r}^{C / B} \times \overline{\mathbf{a}}_{B}}{\rho_{B} l_{B} \mathbf{g}} \ll 1\right)\end{array}$ & Term $(H)=0$ (Eq. 9 simplifies to Eq. 19) & ve, an, ob PIV, AFP \\
\hline 3.5 & $\begin{array}{l}\text { The outer control surface CS is solid and stationary } \\
\qquad(\mathbf{u}=\mathbf{0} \text { at CS })\end{array}$ & $\begin{array}{l}\text { Terms }(\mathrm{D}) \text { and }(\mathrm{I})=0 \text {. If initially tared, term }(\mathrm{F})=0 \text {, and if conditions } 3.1-3.5 \\
\text { all apply, then only Terms }(\mathrm{C}),(\mathrm{E}) \text {, and }(\mathrm{G}) \text { remain (Eqs. } 9 \text { and } 11 \text { simplify to } \\
\text { Eqs. } 20 \text { and } 21 \text { ) }\end{array}$ & ve, an, ob AFP \\
\hline 3.6 & Liquid-gas interface & $\begin{array}{l}\text { The submerged body volume } V_{\mathrm{s}} \text { replaces } V_{B} \text { for condition } 3.3 \text {. Surface tension can } \\
\text { be neglected for large Weber and Bond numbers, or if force is oriented mostly } \\
\text { towards the body's center of mass }\end{array}$ & ve, an AFP \\
\hline 3.7 & Variable body volume & $\begin{array}{l}\text { Condition } 3.2 \text { can only be assumed for small fluid-body density ratios, or if the time } \\
\text { rate of change of the body volume } \dot{V}_{B} \text { is small relative to the body volume } V_{B} \text {, } \\
\text { or the average fluid velocity is near zero }(\overline{\mathbf{u}} \approx 0) \text {, or the average fluid velocity is } \\
\text { aligned with } \mathbf{r} C_{\mathrm{o}} / B \text {, the vector connecting the CV's center of mass to the body's } \\
\qquad\left(\epsilon_{\dot{V}_{B}}=\frac{V_{\mathrm{o}}}{V} \frac{\rho_{\mathrm{f}}}{\rho_{B}} \frac{\dot{V}_{B}}{V_{B}} \frac{\mathbf{r}^{C_{\mathrm{o}} / B} \times \overline{\mathbf{u}}}{l_{B} \mathbf{g}} \ll 1\right)\end{array}$ & ve, an, ob PIV, AFP \\
\hline
\end{tabular}

The simplest moment and force formulations result when the conditions in Sects. 3.1-3.5 (which are listed in the "conditions" column above) all apply. These formulations are relevant for a wide range of applications, including particle image velocimetry (PIV) or aerodynamic/ hydrodynamic force platform (AFP) studies of vehicles (ve), animals (an), and objects (ob) 


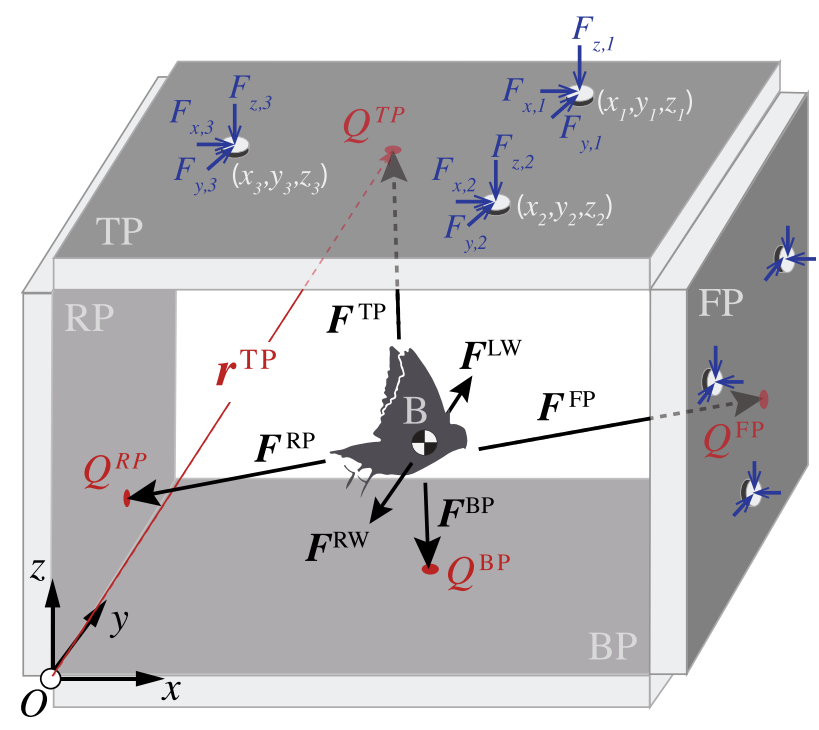

Fig. 2 The net moment on a deforming body (we show a bird as an example) can be recovered from instrumented plates that make up the top (TP), bottom (BP), front (FP), rear (RP), left (LW), and right (RW) walls of the outer control surface of an AFP. The side plates (LW, RW) are not shown for clarity but are otherwise identical. Each plate is instrumented by three 6-axis force/torque sensors (as shown for the top and front plates but not the rear, bottom, and side plates). The sensor forces and torques are used to calculate the net force on each plate $\left(\boldsymbol{F}^{\mathrm{TP}}, \boldsymbol{F}^{\mathrm{FP}}, \boldsymbol{F}^{\mathrm{BP}}, \boldsymbol{F}^{\mathrm{RP}}, \boldsymbol{F}^{\mathrm{LW}}, \boldsymbol{F}^{\mathrm{RW}}\right)$, as well as the position of the center of force (the zero moment point, $Q^{\text {Plate }}$ ) on each plate. Sensor forces and locations are labeled on the top plate as $F_{x, s}, F_{y, s}, F_{z, s}$ and $\left(x_{s}, y_{s}, z_{s}\right)$, where $s=1,2,3$, and $\boldsymbol{r}^{\mathrm{TP}}$ is the position vector from the origin to the center of force on the top plate. We use the same notation for the sensor forces and locations on the other five plates in our formulations (we omit the labels here to improve figure clarity)

were not sacrificed, all training and experimental procedures were approved by Stanford's Administrative Panel on Laboratory Animal Care). We recorded each flight with five DLTcalibrated (Hedrick 2008) high-speed cameras, which were synchronized with each other and the AFP. To estimate the position of a bird's center of gravity, we took a weighted average of the tracked coordinates of the bird's left eye and most distal tip of the tail $\left(x_{\mathrm{cg}}=0.5 x_{\text {eye }}+0.5 x_{\text {tail }}, y_{\mathrm{cg}}=y_{\text {tail }}\right.$, and $z_{\mathrm{cg}}=0.69 z_{\text {eye }}+0.31 z_{\text {tail }}$ based on estimated mass distributions from previous cadaver studies).

During these short $(80 \mathrm{~cm})$ flights, four of the parrotlets often briefly pause their wingbeat after completing their takeoff acceleration and before decelerating for landing. The downstroke that precedes this pause generates mostly vertical weight support (Fig. 3a) while horizontal forces remain relatively low (Fig. 3b). This enables us to more intuitively understand the resulting fluid moment acting about the bird's center of gravity. The first half of the downstroke yields a positive (forward pitching) moment as net fluid forces push upwards on the wings, which are sweeping forward from behind the bird's center of gravity. As the wings sweep in front of the

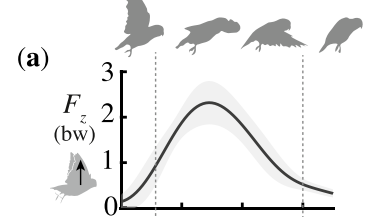

(b)

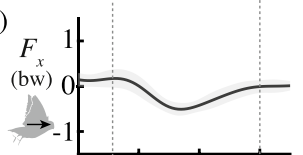

(c)

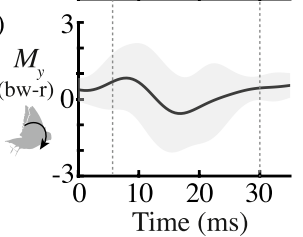

Fig. 3 The fluid moment generated during a bird's downstroke can be derived from the fluid forces measured in an aerodynamic force platform. During a downstroke prior to a wingbeat pause, a bird generates a primarily weight support and $\mathbf{b}$ relatively little force in the forward direction. c By combining these force measurements with our fluid moment formulations, we find that the start of the downstroke yields a small positive moment which is largely canceled out by a small negative moment generated during the second half of the downstroke. Curves and shaded regions show mean $\pm \mathrm{SD}$ for $N=4$ birds, $n=15$ flights. Dashed vertical lines show average downstroke start and end times, and arrows on avatars indicate positive sign conventions. Forces are normalized by bodyweight bw, and the moment about the bird's center of gravity $M_{y}$ is normalized by the bird's bodyweight times its wing radius $r$

bird's center of gravity, the weight support that they generate yields a negative (backward pitching) moment. Because the bird's body orientation remains mostly constant during the pause, we would expect the net fluid moment generated about the bird's center of mass during the preceding downstroke to be near zero. Indeed, integrating the calculated moment (Fig. 3c), which we normalize by the bird's bodyweight bw and mid-downstroke wing radius $r$, we find a net moment impulse during each downstroke of only $0.004 \pm 0.028 \mathrm{bw}$ r-s ( $N=4$ birds, $n=15$ flights). These small fluid moments likely exhibit greater variance compared to the measured forces because they are particularly sensitive to setup noise and leakage effects. Based on the theory presented here, future PIV and AFP studies can build off our first results and further improve the experimental implementation.

\section{Conclusion}

Control surface formulations can enable both direct and nonintrusive measurement of the net fluid force and moment acting on a deforming body. Lentink (2018) previously showed how the Reynolds transport theorem for conservation of momentum can be used to accurately recover the 3D instantaneous forces generated by freely moving deformable bodies using control 
surface integration. We have now demonstrated how this methodology can be extended to recover the resulting 3D moments by applying the Reynolds transport theorem for conservation of angular momentum. In contrast to existing methods that can only be used for tethered or constrained locomotion, this methodology applies to many more forms of locomotion studies, including that of freely moving vehicles, animals, and deforming objects measured using particle image velocimetry and aerodynamic force platforms. For many of these PIV and AFP studies, the formulation for recovering fluid moments about a body's center of mass can be greatly simplified (Table 1). In general, these fluid force and moment control surface formulations provide the potential for researchers to gain a new understanding of how deforming bodies locomote in gas, liquid, or at the liquid-gas interface in science and engineering.

Acknowledgements We thank Karen May Wang for critically reading the manuscript. DL was supported by NSF CAREER Award 1552419. DDC was supported by a National Defense Science and Engineering Graduate Fellowship and a Stanford Graduate Fellowship.

Open Access This article is distributed under the terms of the Creative Commons Attribution 4.0 International License (http://creativeco mmons.org/licenses/by/4.0/), which permits unrestricted use, distribution, and reproduction in any medium, provided you give appropriate credit to the original author(s) and the source, provide a link to the Creative Commons license, and indicate if changes were made.

\section{Appendix}

This Appendix provides the full details for deriving the full fluid moment equation (Eq. 11) beginning from Eq. (4) in Sect. 2 of the main text:

$$
\begin{gathered}
\iiint_{C V} \frac{\partial}{\partial t}(\mathbf{r} \times \mathbf{u}) \rho \mathrm{d} V+\iint_{\partial V}(\mathbf{r} \times \mathbf{u})(\mathbf{u} \cdot \mathbf{n}) \rho \mathrm{d} S \\
=-\iint_{\partial V} \mathbf{r} \times p \mathbf{n} \mathrm{d} S+\iint_{\partial V} \mathbf{r} \times(\overline{\bar{\tau}} \cdot \mathbf{n}) \mathrm{d} S \\
+\iiint_{C V} \mathbf{r} \times \mathbf{g} \rho \mathrm{d} V .
\end{gathered}
$$

For completion, details included in Sect. 2 are repeated below. We begin by assuming a constant density flow to bring the fluid density $\rho$ out of the integrals:

$$
\begin{aligned}
\rho \iiint_{C V} & \frac{\partial}{\partial t}(\mathbf{r} \times \mathbf{u}) \mathrm{d} V+\rho \iint_{\partial V}(\mathbf{r} \times \mathbf{u})(\mathbf{u} \cdot \mathbf{n}) \mathrm{d} S \\
= & -\iint_{\partial V} \mathbf{r} \times p \mathbf{n} \mathrm{d} S \\
& +\iint_{\partial V} \mathbf{r} \times(\overline{\bar{\tau}} \cdot \mathbf{n}) \mathrm{d} S+\rho \iiint_{C V} \mathbf{r} \times \mathbf{g d} V .
\end{aligned}
$$

We next simplify this expression by introducing the position of the control volume's center of mass $C$ relative to $O$, $\mathbf{r}^{C / O}$ (Fig. 1a). We note that while a spatial control volume does not have a center of mass, we can treat CV as a material volume by defining the control volume velocity to be equal to the material velocity at all times (Sonin 2001). As we discuss in Sect. $3, \mathbf{r}^{C / O}$ depends on the position of the body (or more specifically, where the volume displaced by the body is) within the $\mathrm{CV}$ and can, therefore, vary in time. The position vector from the origin $O$ to a mass element $\mathrm{d} m$ located at point $P$ can be expressed in terms of $\mathbf{r}^{C / O}$ as $\mathbf{r}=\mathbf{r}^{P / C}+\mathbf{r}^{C / O}$ (where $\mathbf{r}^{P / C}$ is the position vector from $C$ to $\mathrm{d} m$ ). By the definition of a center of mass, $\rho \iiint_{C V} \mathbf{r}^{P / C} \mathrm{~d} V=\iiint_{C V} \mathbf{r}^{P / C} \mathrm{~d} m=0$. We can thus rewrite the final gravity term as $\rho \iiint_{C V} \mathbf{r} \times \mathbf{g d} V=\rho \iiint_{C V}\left(\mathbf{r}^{P / C}+\mathbf{r}^{C / O}\right)$ $\times \mathbf{g d} V=\rho \iiint_{C V} \mathbf{r}^{C / O} \times \mathbf{g d} V=\mathbf{r}^{C / O} \times \mathbf{g} \rho V$, where $V$ is the fluid volume in the CV. Equation (29) then becomes:

$$
\begin{aligned}
\rho \iiint_{C V} \frac{\partial}{\partial t}(\mathbf{r} \times \mathbf{u}) \mathrm{d} V+\rho \iint_{\partial V}(\mathbf{r} \times \mathbf{u})(\mathbf{u} \cdot \mathbf{n}) \mathrm{d} S \\
=-\iint_{\partial V} \mathbf{r} \times p \mathbf{n} \mathrm{d} S \\
\quad+\iint_{\partial V} \mathbf{r} \times(\overline{\bar{\tau}} \cdot \mathbf{n}) \mathrm{d} S+\mathbf{r}^{C / O} \times \mathbf{g} \rho V .
\end{aligned}
$$

We first reformulate the left-hand side of Eq. (30) into terms that can be more easily evaluated, beginning with the unsteady volume integral. The cross product of a vector with itself is zero, so we start by writing the unsteady term as:

$$
\begin{aligned}
\rho \iiint_{C V} \frac{\partial}{\partial t}(\mathbf{r} \times \mathbf{u}) \mathrm{d} V & =\rho \iiint \int_{C V} \frac{\partial \mathbf{r}}{\partial t} \times \mathbf{u}+\mathbf{r} \times \frac{\partial \mathbf{u}}{\partial t} \mathrm{~d} V \\
& =\rho \iiint \int_{C V} \mathbf{u} \times \mathbf{u}+\mathbf{r} \times \frac{\partial \mathbf{u}}{\partial t} \mathrm{~d} V \\
& =\rho \iiint \int_{C V} \mathbf{r} \times \frac{\partial \mathbf{u}}{\partial t} \mathrm{~d} V .
\end{aligned}
$$

We then expand the integral by leveraging the position and velocity of the control volume's center of mass, $C$. Just as the position vector of a fluid element at $P$ could be expressed as $\mathbf{r}=\mathbf{r}^{P / C}+\mathbf{r}^{C / O}$, the velocity of the fluid element can be written as $\mathbf{u}=\mathbf{u}^{P / C}+\mathbf{u}^{C / O}$, where $\mathbf{u}^{P / C}$ is the fluid element's velocity relative to $C$, and $\mathbf{u}^{C / O}$ is the velocity of $C$ relative to $O$ :

$$
\begin{aligned}
\rho \iiint_{C V} \mathbf{r} \times \frac{\partial \mathbf{u}}{\partial t} \mathrm{~d} V \\
=\rho \iiint_{C V}\left(\mathbf{r}^{P / C}+\mathbf{r}^{C / O}\right) \times \frac{\partial}{\partial t}\left(\mathbf{u}^{P / C}+\mathbf{u}^{C / O}\right) \mathrm{d} V \\
=\rho \iiint_{C V} \mathbf{r}^{P / C} \times \frac{\partial \mathbf{u}^{P / C}}{\partial t}+\mathbf{r}^{P / C} \\
\quad \times \frac{\partial \mathbf{u}^{C / O}}{\partial t}+\mathbf{r}^{C / O} \times\left(\frac{\partial \mathbf{u}^{P / C}}{\partial t}+\frac{\partial \mathbf{u}^{C / O}}{\partial t}\right) \mathrm{d} V \\
=\rho \iiint_{C V} \mathbf{r}^{P / C} \times \frac{\partial \mathbf{u}^{P / C}}{\partial t} \mathrm{~d} V+\rho \iiint_{C V} \mathbf{r}^{P / C} \\
\quad \times \frac{\partial \mathbf{u}^{C / O}}{\partial t} \mathrm{~d} V+\rho \iiint_{C V} \mathbf{r}^{C / O} \times \frac{\partial \mathbf{u}}{\partial t} \mathrm{~d} V
\end{aligned}
$$


and bringing $\frac{\partial \mathbf{u}^{C / O}}{\partial t}$ and $\mathbf{r}^{C / O}$ out of the integrals because they are the same for all fluid elements in the CV (they are specific only to $C$ ),

$$
\begin{aligned}
= & \rho \iiint_{C V} \mathbf{r}^{P / C} \times \frac{\partial \mathbf{u}^{P / C}}{\partial t} \mathrm{~d} V+\rho \iiint_{C V} \mathbf{r}^{P / C} \mathrm{~d} V \\
& \times \frac{\partial \mathbf{u}^{C / O}}{\partial t}+\mathbf{r}^{C / O} \times \rho \iiint_{C V} \frac{\partial \mathbf{u}}{\partial t} \mathrm{~d} V .
\end{aligned}
$$

We can now again take advantage of the definition of center of mass $\left(\rho \iiint_{C V} \mathbf{r}^{P / C} \mathrm{~d} V=0\right)$ to eliminate the second term. We can also rewrite the last partial time derivative in terms of a material time derivative and surface integral (see Eq. 1 from Sect. 2 with $\mathrm{CM}=\rho C V$ ), which will enable us to combine it with the convective term from Eq. (30):

$$
\begin{aligned}
& \rho \iiint_{C V} \mathbf{r}^{P / C} \times \frac{\partial \mathbf{u}^{P / C}}{\partial t} \mathrm{~d} V+0+\mathbf{r}^{C / O} \times \rho \iiint_{C V} \frac{\partial \mathbf{u}}{\partial t} \mathrm{~d} V \\
& =\rho \iiint_{C V} \mathbf{r}^{P / C} \times \frac{\partial \mathbf{u}^{P / C}}{\partial t} \mathrm{~d} V \\
& \quad+\mathbf{r}^{C / O} \times \rho\left[\frac{\mathrm{d}}{\mathrm{d} t} \iiint_{C V} \mathbf{u d} V-\iint_{\partial V} \mathbf{u}(\mathbf{u} \cdot \mathbf{n}) \mathrm{d} S\right] \\
& =\rho \iiint_{C V} \mathbf{r}^{P / C} \times \frac{\partial \mathbf{u}^{P / C}}{\partial t} \mathrm{~d} V \\
& \quad+\mathbf{r}^{C / O} \times \rho \frac{\mathrm{d}}{\mathrm{d} t} \iiint \int_{C V} \mathbf{u d} V-\mathbf{r}^{C / O} \times \rho \iint_{\partial V} \mathbf{u}(\mathbf{u} \cdot \mathbf{n}) \mathrm{d} S \\
& =\rho \iiint_{C V} \mathbf{r}^{P / C} \times \frac{\partial \mathbf{u}^{P / C}}{\partial t} \mathrm{~d} V+\mathbf{r}^{C / O} \times \rho \frac{\mathrm{d}}{\mathrm{d} t} \iiint_{C V} \mathbf{u d} V \\
& \quad-\rho \iint_{\partial V}\left(\mathbf{r}^{C / O} \times \mathbf{u}\right)(\mathbf{u} \cdot \mathbf{n}) \mathrm{d} S .
\end{aligned}
$$

We can now add in the convective term to complete our new expression for the left side of Eq. (30):

$$
\begin{aligned}
& \rho \iiint_{C V} \mathbf{r}^{P / C} \times \frac{\partial \mathbf{u}^{P / C}}{\partial t} \mathrm{~d} V+\mathbf{r}^{C / O} \\
& \quad \times \rho \frac{\mathrm{d}}{\mathrm{d} t} \iiint_{C V} \mathbf{u} \mathrm{d} V-\rho \iint_{\partial V}\left(\mathbf{r}^{C / O} \times \mathbf{u}\right)(\mathbf{u} \cdot \mathbf{n}) \mathrm{d} S \\
& \quad+\rho \iint_{\partial V}(\mathbf{r} \times \mathbf{u})(\mathbf{u} \cdot \mathbf{n}) \mathrm{d} S \\
& =\rho \iiint_{C V} \mathbf{r}^{P / C} \times \frac{\partial \mathbf{u}^{P / C}}{\partial t} \mathrm{~d} V+\mathbf{r}^{C / O} \times \rho \frac{\mathrm{d}}{\mathrm{d} t} \iiint_{C V} \mathbf{u d} V \\
& \quad+\rho \iint_{\partial V}\left(\left(\mathbf{r}-\mathbf{r}^{C / O}\right) \times \mathbf{u}\right)(\mathbf{u} \cdot \mathbf{n}) \mathrm{d} S \\
& =\rho \iiint_{C V} \mathbf{r}^{P / C} \times \frac{\partial \mathbf{u}^{P / C}}{\partial t} \mathrm{~d} V+\mathbf{r}^{C / O} \\
& \quad \times \rho \frac{\mathrm{d}}{\mathrm{d} t} \iiint_{C V} \mathbf{u d} V+\rho \iint_{\partial V}\left(\mathbf{r}^{P / C} \times \mathbf{u}\right)(\mathbf{u} \cdot \mathbf{n}) \mathrm{d} S .
\end{aligned}
$$

Before we add in the right-hand side of Eq. (30), we will apply a few more reformulations to express the integral terms in Eq. (32) (i.e. the left-hand side of Eq. 30) more intuitively. Beginning with the first term, we apply the product rule and the fact that the cross product of a vector with itself is zero:

$$
\begin{aligned}
& \rho \iiint_{C V} \mathbf{r}^{P / C} \times \frac{\partial \mathbf{u}^{P / C}}{\partial t} \mathrm{~d} V \\
& \quad=\rho \iiint_{C V} \frac{\partial}{\partial t}\left(\mathbf{r}^{P / C} \times \mathbf{u}^{P / C}\right)-\left(\frac{\partial \mathbf{r}^{P / C}}{\partial t} \times \mathbf{u}^{P / C}\right) \mathrm{d} V \\
& \quad=\rho \iiint_{C V} \frac{\partial}{\partial t}\left(\mathbf{r}^{P / C} \times \mathbf{u}^{P / C}\right) \mathrm{d} V .
\end{aligned}
$$

We can also expand the third term in Eq. (32) into two surface integrals (again using $\mathbf{u}=\mathbf{u}^{P / C}+\mathbf{u}^{C / O}$ ), which will enable us to regroup the terms in Eq. (32) with another material time derivative:

$$
\begin{aligned}
& \rho \iint_{\partial V}\left(\mathbf{r}^{P / C} \times \mathbf{u}\right)(\mathbf{u} \cdot \mathbf{n}) \mathrm{d} S \\
& =\rho \iint_{\partial V}\left(\mathbf{r}^{P / C} \times \mathbf{u}^{P / C}\right)(\mathbf{u} \cdot \mathbf{n}) \mathrm{d} S \\
& \quad+\rho \iint_{\partial V}\left(\mathbf{r}^{P / C} \times \mathbf{u}^{C / O}\right)(\mathbf{u} \cdot \mathbf{n}) \mathrm{d} S .
\end{aligned}
$$

We can now rewrite the terms from Eq. (32) as

$$
\begin{aligned}
& \rho \iiint_{C V} \frac{\partial}{\partial t}\left(\mathbf{r}^{P / C} \times \mathbf{u}^{P / C}\right) \mathrm{d} V \\
& \quad+\mathbf{r}^{C / O} \times \rho \frac{\mathrm{d}}{\mathrm{d} t} \iiint_{C V} \mathbf{u d} V \\
& \quad+\rho \iint_{\partial V}\left(\mathbf{r}^{P / C} \times \mathbf{u}^{P / C}\right)(\mathbf{u} \cdot \mathbf{n}) \mathrm{d} S \\
& \quad+\rho \iint_{\partial V}\left(\mathbf{r}^{P / C} \times \mathbf{u}^{C / O}\right)(\mathbf{u} \cdot \mathbf{n}) \mathrm{d} S,
\end{aligned}
$$

or rearranging,

$$
\begin{aligned}
& =\rho \iiint_{C V} \frac{\partial}{\partial t}\left(\mathbf{r}^{P / C} \times \mathbf{u}^{P / C}\right) \mathrm{d} V \\
& +\rho \iint_{\partial V}\left(\mathbf{r}^{P / C} \times \mathbf{u}^{P / C}\right)(\mathbf{u} \cdot \mathbf{n}) \mathrm{d} S \\
& +\rho \iint_{\partial V}\left(\mathbf{r}^{P / C} \times \mathbf{u}^{C / O}\right)(\mathbf{u} \cdot \mathbf{n}) \mathrm{d} S \\
& +\mathbf{r}^{C / O} \times \rho \frac{\mathrm{d}}{\mathrm{d} t} \iiint_{C V} \mathbf{u d} V .
\end{aligned}
$$

We see that the first two integrals are equal to the material time derivative, so Eq. (32) can be written as: 


$$
\begin{gathered}
=\underbrace{\rho \frac{\mathrm{d}}{\mathrm{d} t} \iiint_{C V}\left(\mathbf{r}^{P / C} \times \mathbf{u}^{P / C}\right) \mathrm{d} V}_{\text {I }} \\
+\underbrace{\rho \iint_{\partial V}\left(\mathbf{r}^{P / C} \times \mathbf{u}^{C / O}\right)(\mathbf{u} \cdot \mathbf{n}) \mathrm{d} S}_{\mathrm{II}} \\
+\underbrace{\mathbf{r}^{C / O} \times \rho \frac{\mathrm{d}}{\mathrm{d} t} \iiint_{C V} \mathbf{u d} V}_{\mathrm{III}} .
\end{gathered}
$$

The first time derivative in Eq. (33) (I) is the moment of CV about its center of mass $C$,

$\rho \frac{\mathrm{d}}{\mathrm{d} t} \iiint_{C V}\left(\mathbf{r}^{P / C} \times \mathbf{u}^{P / C}\right) \mathrm{d} V=\frac{\mathrm{d}}{\mathrm{d} t} \mathbf{H}^{\mathrm{CV} / C}=\mathbf{M}^{\mathrm{CV} / C}$.

The second term (II) can be simplified if we leverage the definition of center of mass again. In order to do so, we first apply the Reynolds transport theorem (Eq. 29):

$$
\begin{aligned}
& \rho \iint_{\partial V}\left(\mathbf{r}^{P / C} \times \mathbf{u}^{C / O}\right)(\mathbf{u} \cdot \mathbf{n}) \mathrm{d} S \\
& =\rho \frac{\mathrm{d}}{\mathrm{d} t} \iiint_{C V}\left(\mathbf{r}^{P / C} \times \mathbf{u}^{C / O}\right) \mathrm{d} V \\
& \quad-\rho \iiint_{C V} \frac{\partial}{\partial t}\left(\mathbf{r}^{P / C} \times \mathbf{u}^{C / O}\right) \mathrm{d} V \\
& =\rho \frac{\mathrm{d}}{\mathrm{d} t} \iiint_{C V}\left(\mathbf{r}^{P / C} \times \mathbf{u}^{C / O}\right) \mathrm{d} V \\
& \quad-\rho \iiint_{C V}\left(\frac{\partial \mathbf{r}^{P / C}}{\partial t} \times \mathbf{u}^{C / O}+\mathbf{r}^{P / C} \times \frac{\partial \mathbf{u}^{C / O}}{\partial t}\right) \mathrm{d} V \\
& =\rho \frac{\mathrm{d}}{\mathrm{d} t} \iiint_{C V}\left(\mathbf{r}^{P / C} \times \mathbf{u}^{C / O}\right) \mathrm{d} V \\
& \quad-\rho \iint_{C V} \frac{\partial \mathbf{r}^{P / C}}{\partial t} \times \mathbf{u}^{C / O} \mathrm{~d} V \\
& \quad-\rho \iiint_{C V} \mathbf{r}^{P / C} \times \frac{\partial \mathbf{u}^{C / O}}{\partial t} \mathrm{~d} V .
\end{aligned}
$$

Then pulling the terms specific only to $C\left(\mathbf{u}^{C / O}\right.$ and $\left.\frac{\partial \mathbf{u}^{C / O}}{\partial t}\right)$ out of the integrals,

$$
\begin{gathered}
=\frac{\mathrm{d}}{\mathrm{d} t}\left(\rho \iiint \int_{C V} \mathbf{r}^{P / C} \mathrm{~d} V \times \mathbf{u}^{C / O}\right) \\
-\rho \iiint_{C V} \frac{\partial \mathbf{r}^{P / C}}{\partial t} \mathrm{~d} V \times \mathbf{u}^{C / O} \\
-\rho \iiint_{C V} \mathbf{r}^{P / C} \mathrm{~d} V \times \frac{\partial \mathbf{u}^{C / O}}{\partial t}
\end{gathered}
$$

we can now utilize the center of mass definition, $\rho \iiint_{C V} \mathbf{r}^{P / C} \mathrm{~d} V=0$, to eliminate the first and last terms, which leaves us with: $=-\rho \iiint_{C V} \frac{\partial \mathbf{r}^{P / C}}{\partial t} \mathrm{~d} V \times \mathbf{u}^{C / O}$.

Then since $\frac{\partial \mathbf{r}^{P / C}}{\partial t}=\mathbf{u}^{P / C}=\mathbf{u}^{P / O}-\mathbf{u}^{C / O}$, and again using the fact that the cross product of a vector with itself is zero, term II becomes:

$$
\begin{aligned}
& -\rho \iiint \int_{C V}\left(\mathbf{u}^{P / O}-\mathbf{u}^{C / O}\right) \mathrm{d} V \times \mathbf{u}^{C / O} \\
& =-\rho \iiint \int_{C V}\left(\mathbf{u}^{P / O} \times \mathbf{u}^{C / O}-\mathbf{u}^{C / O} \times \mathbf{u}^{C / O}\right) \mathrm{d} V \\
& =-\rho \iiint_{C V} \mathbf{u}^{P / O} \times \mathbf{u}^{C / O} \mathrm{~d} V \\
& =-\rho \iiint_{C V} \mathbf{u}^{P / O} \mathrm{~d} V \times \mathbf{u}^{C / O} \\
& =\mathbf{u}^{C / O} \times \rho \iiint_{C V} \mathbf{u} \mathrm{d} V .
\end{aligned}
$$

Our ultimate goal is to develop a control surface formulation, so we next convert the volume integral into a surface integral. For brevity, we temporarily switch to Einstein notation to show the steps involved in this transformation:

$$
\begin{aligned}
\iiint_{C V} \mathbf{u} \mathrm{d} V & =\iiint_{C V} u_{i} \mathrm{~d} V=\iiint_{C V} \frac{\partial x_{i}}{\partial x_{j}} u_{j} \mathrm{~d} V \\
& =\iiint_{C V} \frac{\partial}{\partial x_{j}}\left(x_{i} u_{j}\right) \mathrm{d} V-\iiint_{C V} x_{i} \frac{\partial u_{j}}{\partial x_{j}} \mathrm{~d} V .
\end{aligned}
$$

Applying Gauss's theorem to the first term and using the fact that the divergence $\frac{\partial u_{j}}{\partial x_{j}}=0$ for incompressible flow to eliminate the second term,

$\iiint_{C V} \mathbf{u d} V=\iint_{\partial V} x_{i} u_{j} n_{j} \mathrm{~d} S=\iint_{\partial V} \mathbf{r}(\mathbf{u} \cdot \mathbf{n}) \mathrm{d} S$.

Term (II) thus becomes:

$\mathbf{u}^{C / O} \times \rho \iiint_{C V} \mathbf{u} \mathrm{d} V=\mathbf{u}^{C / O} \times \rho \iint_{\partial V} \mathbf{r}(\mathbf{u} \cdot \mathbf{n}) \mathrm{d} S$

To express the third term (III) from Eq. (33) in terms of surface integrals, we can apply the conservation of momentum:

$$
\begin{aligned}
\mathbf{r}^{C / O} & \times \rho \frac{\mathrm{d}}{\mathrm{d} t} \iiint_{C V} \mathbf{u d} V \\
= & \mathbf{r}^{C / O} \times\left(-\iint_{\partial V} p \mathbf{n} \mathrm{d} S+\iint_{\partial V}(\overline{\bar{\tau}} \cdot \mathbf{n}) \mathrm{d} S\right. \\
& \left.-\rho \iint_{\partial V} \mathbf{u}((\mathbf{u}-\mathbf{v}) \cdot \mathbf{n}) \mathrm{d} S\right)
\end{aligned}
$$

where $\mathbf{v}$ is the velocity of the control surface.

We can now combine the simplified forms of (I)-(III) from Eqs. (34)-(36) with the right-hand side of Eq. (30) to form the full moment equation: 


$$
\begin{aligned}
\mathbf{M}^{\mathrm{CV} / C}+\mathbf{u}^{C / O} \times \rho \iint_{\partial V} \mathbf{r}(\mathbf{u} \cdot \mathbf{n}) \mathrm{d} S \\
+\mathbf{r}^{C / O} \times\left(-\iint_{\partial V} p \mathbf{n} \mathrm{d} S+\iint_{\partial V}(\overline{\bar{\tau}} \cdot \mathbf{n}) \mathrm{d} S\right. \\
\left.-\rho \iint_{\partial V} \mathbf{u}((\mathbf{u}-\mathbf{v}) \cdot \mathbf{n})\right) \mathrm{d} S \\
=-\iint_{\partial V} \mathbf{r} \times p \mathbf{n} \mathrm{d} S \\
+\iint_{\partial V} \mathbf{r} \times(\overline{\bar{\tau}} \cdot \mathbf{n}) \mathrm{d} S+\mathbf{r}^{C / O} \times \mathbf{g} \rho V .
\end{aligned}
$$

Next, to isolate fluid moments acting on the body from the rest of the control mass, we follow Lentink (2018) and consider the continuous control surface $\partial V$ in terms of the outer control surface $\mathrm{CS}$, the inner control surface that encloses the deforming body $\partial B$, and a infinitesimally thin tube that connects the body and outer surface $\partial b$, i.e. control surface $(\partial V)=$ outer surface $(\mathrm{CS})+$ tube $(\partial b)+$ body surface $(\partial B)$ (Fig. 1a). We can, therefore, expand the control surface terms:

$$
\begin{aligned}
& \mathbf{M}^{\mathrm{CV} / C}+\mathbf{u}^{\mathrm{C} / O} \\
& \times \rho\left(\iint_{\mathrm{CS}} \mathbf{r}(\mathbf{u} \cdot \mathbf{n}) \mathrm{d} S+\iint_{\partial b} \mathbf{r}(\mathbf{u} \cdot \mathbf{n}) \mathrm{d} S\right. \\
& \left.+\iint_{\partial B} \mathbf{r}(\mathbf{u} \cdot \mathbf{n}) \mathrm{d} S\right) \\
& +\mathbf{r}^{C / O} \times\left(-\iint_{\mathrm{CS}} p \mathbf{n d} S+\iint_{\mathrm{CS}}(\overline{\bar{\tau}} \cdot \mathbf{n}) \mathrm{d} S\right. \\
& \left.-\rho \iint_{\mathrm{CS}} \mathbf{u}((\mathbf{u}-\mathbf{v}) \cdot \mathbf{n})\right) \mathrm{d} S \\
& -\iint_{\partial b} p \mathbf{n} \mathrm{d} S+\iint_{\partial b}(\overline{\bar{\tau}} \cdot \mathbf{n}) \mathrm{d} S \\
& \left.-\rho \iint_{\partial b} \mathbf{u}((\mathbf{u}-\mathbf{v}) \cdot \mathbf{n})\right) \mathrm{d} S \\
& -\iint_{\partial B} p \mathbf{n d} S+\iint_{\partial B}(\overline{\bar{\tau}} \cdot \mathbf{n}) \mathrm{d} S \\
& \left.-\rho \iint_{\partial B} \mathbf{u}((\mathbf{u}-\mathbf{v}) \cdot \mathbf{n})\right) \mathrm{d} S \\
& =-\iint_{\mathrm{CS}} \mathbf{r} \times p \mathbf{n d} S+\iint_{\mathrm{CS}} \mathbf{r} \times(\overline{\bar{\tau}} \cdot \mathbf{n}) \mathrm{d} S \\
& -\iint_{\partial b} \mathbf{r} \times p \mathbf{n d} S+\iint_{\partial b} \mathbf{r} \times(\overline{\bar{\tau}} \cdot \mathbf{n}) \mathrm{d} S \\
& -\iint_{\partial B} \mathbf{r} \times p \mathbf{n} \mathrm{d} S+\iint_{\partial B} \mathbf{r} \times(\overline{\bar{\tau}} \cdot \mathbf{n}) \mathrm{d} S \\
& +\mathbf{r}^{C / O} \times \mathbf{g} \rho V .
\end{aligned}
$$

Moments on opposite sides of the infinitesimal tube $\partial b$ are equal and opposite and, therefore, cancel out (so all $\partial b$ integrals go to zero), and the convective term vanishes on the body surface because $\mathbf{u}-\mathbf{v}=0$ due to the no-flow boundary condition:

$$
\begin{aligned}
& \mathbf{M}^{\mathrm{CV} / C}+\mathbf{u}^{C / O} \times \rho\left(\iint_{\mathrm{CS}} \mathbf{r}(\mathbf{u} \cdot \mathbf{n}) \mathrm{d} S+\iint_{\partial B} \mathbf{r}(\mathbf{u} \cdot \mathbf{n}) \mathrm{d} S\right) \\
& +\mathbf{r}^{C / O} \times\left(-\iint_{\mathrm{CS}} p \mathbf{n d} S+\iint_{\mathrm{CS}}(\overline{\bar{\tau}} \cdot \mathbf{n}) \mathrm{d} S\right. \\
& -\rho \iint_{\mathrm{CS}} \mathbf{u}((\mathbf{u}-\mathbf{v}) \cdot \mathbf{n}) \mathrm{d} S \\
& \left.-\iint_{\partial B} p \mathbf{n d} S+\iint_{\partial B}(\overline{\bar{\tau}} \cdot \mathbf{n}) \mathrm{d} S\right) \\
& =-\iint_{\mathrm{CS}} \mathbf{r} \times p \mathbf{n} \mathrm{d} S+\iint_{\mathrm{CS}} \mathbf{r} \times(\overline{\bar{\tau}} \cdot \mathbf{n}) \mathrm{d} S \\
& \underbrace{-\iint_{\partial B} \mathbf{r} \times p \mathbf{n d} S+\iint_{\partial B} \mathbf{r} \times(\overline{\bar{\tau}} \cdot \mathbf{n}) \mathrm{d} S}_{\text {net pressure and shear torque from body }}+\mathbf{r}^{C / O} \times \mathbf{g} \rho V .
\end{aligned}
$$

Using this new formulation, we can now derive the total moment acting on the body. The moment caused by pressure and shear on the surface cutout around the body $(\partial B)$ represents the net moment from the body on the fluid. The moment on the body from the fluid will, therefore, be equal and opposite. To find the total external moment on the body with respect to the origin, $\mathbf{M}^{O}$, we must also add in the moment caused by gravity. For a body with mass $m$ and center of mass position $\mathbf{r}^{B / O}$ with respect to the origin (Fig. 1b),

$$
\begin{aligned}
\mathbf{M}^{O}= & -\left(-\iint_{\partial B} \mathbf{r} \times p \mathbf{n} \mathrm{d} S+\iint_{\partial B} \mathbf{r} \times(\overline{\bar{\tau}} \cdot \mathbf{n}) \mathrm{d} S\right) \\
& +\mathbf{r}^{B / O} \times m \mathbf{g} .
\end{aligned}
$$

We can solve for $\mathbf{M}^{O}$ using Eq. (38):

$$
\begin{aligned}
\mathbf{M}^{O}= & -\mathbf{M}^{\mathrm{CV} / C}-\mathbf{u}^{C / O} \\
& \times \rho\left(\iint_{\mathrm{CS}} \mathbf{r}(\mathbf{u} \cdot \mathbf{n}) \mathrm{d} S+\iint_{\partial B} \mathbf{r}(\mathbf{u} \cdot \mathbf{n}) \mathrm{d} S\right) \\
& -\mathbf{r}^{C / O} \times\left(-\iint_{\mathrm{CS}} p \mathbf{n} \mathrm{d} S+\iint_{\mathrm{CS}}(\overline{\bar{\tau}} \cdot \mathbf{n}) \mathrm{d} S\right. \\
& -\rho \iint_{\mathrm{CS}} \mathbf{u}((\mathbf{u}-\mathbf{v}) \cdot \mathbf{n}) \mathrm{d} S \\
& \left.-\iint_{\partial B} p \mathbf{n} \mathrm{d} S+\iint_{\partial B}(\overline{\bar{\tau}} \cdot \mathbf{n}) \mathrm{d} S\right) \\
& -\iint_{\mathrm{CS}} \mathbf{r} \times p \mathbf{n} \mathrm{d} S \\
& +\iint_{\mathrm{CS}} \mathbf{r} \times(\overline{\bar{\tau}} \cdot \mathbf{n}) \mathrm{d} S+\mathbf{r}^{C / O} \times \mathbf{g} \rho V+\mathbf{r}^{B / O} \times m \mathbf{g} .
\end{aligned}
$$

Distributing $\mathbf{r}^{C / O}$, 


$$
\begin{aligned}
\mathbf{M}^{O}= & -\mathbf{M}^{\mathrm{CV} / C}-\mathbf{u}^{C / O} \\
& \times \rho\left(\iint_{\mathrm{CS}} \mathbf{r}(\mathbf{u} \cdot \mathbf{n}) \mathrm{d} S+\iint_{\partial B} \mathbf{r}(\mathbf{u} \cdot \mathbf{n}) \mathrm{d} S\right) \\
& -\iint_{\mathrm{CS}}-\mathbf{r}^{C / O} \\
& \times p \mathbf{n} \mathrm{d} S+\iint_{\mathrm{CS}}-\mathbf{r}^{C / O} \times(\overline{\bar{\tau}} \cdot \mathbf{n}) \mathrm{d} S+\mathbf{r}^{C / O} \\
& \times \rho \iint_{\mathrm{CS}} \mathbf{u}((\mathbf{u}-\mathbf{v}) \cdot \mathbf{n}) \mathrm{d} S \\
& -\mathbf{r}^{C / O} \times\left(-\iint_{\partial B} p \mathbf{n} \mathrm{d} S+\iint_{\partial B}(\overline{\bar{\tau}} \cdot \mathbf{n}) \mathrm{d} S\right) \\
& -\iint_{\mathrm{CS}} \mathbf{r} \times p \mathbf{n} \mathrm{d} S+\iint_{\mathrm{CS}} \mathbf{r} \times(\overline{\bar{\tau}} \cdot \mathbf{n}) \mathrm{d} S \\
& +\mathbf{r}^{C / O} \times \mathbf{g} \rho V+\mathbf{r}^{B / O} \times m \mathbf{g},
\end{aligned}
$$

and then grouping the surface integrals for CS:

$$
\begin{aligned}
\mathbf{M}^{O}= & -\mathbf{M}^{\mathrm{CV} / C}-\mathbf{u}^{C / O} \\
& \times \rho\left(\iint_{\mathrm{CS}} \mathbf{r}(\mathbf{u} \cdot \mathbf{n}) \mathrm{d} S+\iint_{\partial B} \mathbf{r}(\mathbf{u} \cdot \mathbf{n}) \mathrm{d} S\right) \\
& -\iint_{\mathrm{CS}}\left(\mathbf{r}-\mathbf{r}^{C / O}\right) \times p \mathbf{n} \mathrm{d} S \\
& +\iint_{\mathrm{CS}}\left(\mathbf{r}-\mathbf{r}^{C / O}\right) \times(\overline{\bar{\tau}} \cdot \mathbf{n}) \mathrm{d} S+\mathbf{r}^{C / O} \\
& \times \rho \iint_{\mathrm{CS}}^{\mathbf{u}((\mathbf{u}-\mathbf{v}) \cdot \mathbf{n}) \mathrm{d} S} \\
& -\mathbf{r}^{C / O} \times(\underbrace{-\iint_{\partial B} p \mathbf{n} \mathrm{d} S+\iint_{\partial B}(\overline{\bar{\tau}} \cdot \mathbf{n}) \mathrm{d} S}_{\text {Net pressure and shear force from body }}) \\
& +\mathbf{r}^{C / O} \times \mathbf{g} \rho V+\mathbf{r}^{B / O} \times m \mathbf{g} .
\end{aligned}
$$

To further simplify this equation, we can replace the body surface pressure and shear integrals by the net force that they have on the fluid in the $\mathrm{CV}$, which is equal and opposite to the net fluid force acting on the body, $\mathbf{F}=-\left(-\iint_{\partial B} p \mathbf{n} \mathrm{d} S+\iint_{\partial B}(\overline{\bar{\tau}} \cdot \mathbf{n}) \mathrm{d} S\right):$

$$
\begin{aligned}
\mathbf{M}^{O}= & -\mathbf{M}^{\mathrm{CV} / C}-\mathbf{u}^{C / O} \\
& \times \rho\left(\iint_{\mathrm{CS}} \mathbf{r}(\mathbf{u} \cdot \mathbf{n}) \mathrm{d} S+\iint_{\partial B} \mathbf{r}(\mathbf{u} \cdot \mathbf{n}) \mathrm{d} S\right) \\
& -\iint_{\mathrm{CS}}\left(\mathbf{r}-\mathbf{r}^{C / O}\right) \times p \mathbf{n} \mathrm{d} S \\
& +\iint_{\mathrm{CS}}\left(\mathbf{r}-\mathbf{r}^{C / O}\right) \times(\overline{\bar{\tau}} \cdot \mathbf{n}) \mathrm{d} S \\
& +\mathbf{r}^{C / O} \times \rho \iint_{\mathrm{CS}} \mathbf{u}((\mathbf{u}-\mathbf{v}) \cdot \mathbf{n}) \mathrm{d} S \\
& +\mathbf{r}^{C / O} \times \mathbf{F}+\mathbf{r}^{C / O} \times \mathbf{g} \rho V+\mathbf{r}^{B / O} \times m \mathbf{g},
\end{aligned}
$$

where the net fluid force on the body $\mathbf{F}$ is given by (Lentink 2018):

$$
\begin{aligned}
\mathbf{F}= & -\iint_{\mathrm{CS}} p \mathbf{n} \mathrm{d} S+\iint_{\mathrm{CS}}(\overline{\bar{\tau}} \cdot \mathbf{n}) \mathrm{d} S \\
& -\rho \iint_{\mathrm{CS}} \mathbf{u}((\mathbf{u}-\mathbf{v}) \cdot \mathbf{n}) \\
& -\underbrace{\rho \frac{\mathrm{d}}{\mathrm{d} t} \iint_{\partial B} \mathbf{r}(\mathbf{u} \cdot \mathbf{n}) \mathrm{d} S}_{\text {unsteady body force, } \mathbf{U B F}}-\rho \frac{\mathrm{d}}{\mathrm{d} t} \iint_{\mathrm{CS}} \mathbf{r}(\mathbf{u} \cdot \mathbf{n}) \mathrm{d} S .
\end{aligned}
$$

A formulation for the moment on a body about its center of mass $B$, rather than about a theoretical origin, would be more physically meaningful and useful for interpreting the body's rotational dynamics and stability. We, therefore, take one final step to apply the shift theorem for the moment of a set of forces (Mitiguy 2015), which enables us to find the moment on the body about $B$ based on the moment on the body about the origin $\mathbf{M}^{O}$, the position of the body's center of mass relative to the origin $\mathbf{r}^{B / O}$, and the net force on the body $\mathbf{F}^{B}$, which includes the net fluid and gravitational forces on the body (Fig. 1b):

$$
\begin{aligned}
\mathbf{M}^{B} & =\mathbf{M}^{O}-\mathbf{r}^{B / O} \times \mathbf{F}^{B}=\mathbf{M}^{O}-\mathbf{r}^{B / O} \times(\mathbf{F}+m \mathbf{g}) \\
& =\mathbf{M}^{O}-\mathbf{r}^{B / O} \times \mathbf{F}-\mathbf{r}^{B / O} \times m \mathbf{g} .
\end{aligned}
$$

From Eqs. (39) and (41), we arrive at the general expression for the moment on the body with respect to its center of mass (Fig. 1c):

$$
\begin{aligned}
\mathbf{M}^{B}= & -\mathbf{M}^{C V / C}-\mathbf{u}^{C / O} \times \rho\left(\iint_{C S} \mathbf{r}(\mathbf{u} \cdot \mathbf{n}) d S+\iint_{\partial B} \mathbf{r}(\mathbf{u} \cdot \mathbf{n}) d S\right) \\
& -\iint_{C S}\left(\mathbf{r}-\mathbf{r}^{C / O}\right) \times p \mathbf{n} d S+\iint_{C S}\left(\mathbf{r}-\mathbf{r}^{C / O}\right) \times(\overline{\bar{\tau}} \cdot \mathbf{n}) d S \\
& +\mathbf{r}^{C / O} \times \rho \iint_{C S} \mathbf{u}((\mathbf{u}-\mathbf{v}) \cdot \mathbf{n}) d S \\
& +\left(\mathbf{r}^{C / O}-\mathbf{r}^{B / O}\right) \times \mathbf{F}+\mathbf{r}^{C / O} \times \mathbf{g} \rho V .
\end{aligned}
$$

\section{References}

Ahn AN, Furrow E, Biewener AA (2004) Walking and running in the red-legged running frog, Kassina maculata. J Exp Biol 207(3):399-410. https://doi.org/10.1242/jeb.00761

Anand M, Seipel J (2019) The rotary component of leg force during walking and running. J R Soc Interface 16:20190105

Arndt A, Brüggemann GP, Virmavirta M, Komi P (1995) Techniques used by Olympic ski jumpers in the transition from takeoff to early flight. J Appl Biomech 11(2):224-237. https://doi.org/10.1123/ jab.11.2.224

Azuma A, Watanabe T (1988) Flight performance of a dragonfly. J Exp Biol 137:221-252

Bahlman JW, Swartz SM, Breuer KS (2013) Design and characterization of a multi-articulated robotic bat wing. Bioinspiration Bio$\operatorname{mim}$ 8(1):016009. https://doi.org/10.1088/1748-3182/8/1/016009 
Baker R (2007) The history of gait analysis before the advent of modern computers. Gait Posture 26(3):331-342. https://doi.org/10.1016/j. gaitpost.2006.10.014

Biewener AA (1998) Muscle-tendon stresses and elastic energy storage during locomotion in the horse. Comp Biochem Physiol Part B Biochem Mol Biol 120(1):73-87. https://doi.org/10.1016/S0305 -0491(98)00024-8

Biewener AA, Blickhan R, Perry AK, Heglund NC, Taylor CR (1988) Muscle forces during locomotion in kangaroo rats: force platform and tendon buckle measurements compared. J Exp Biol 137(1):191-205

Bush J, Hu D (2006) Walking on water: biolocomotion at the interface. Annu Rev Fluid Mech 38:339-369

Cheng B, Deng X, Hedrick TL (2011) The mechanics and control of pitching manoeuvres in a freely flying hawkmoth (Manduca sexta). J Exp Biol 214(24):4092-4106. https://doi.org/10.1242/ jeb.062760

Chin DD, Lentink D (2017) How birds direct impulse to minimize the energetic cost of foraging flight. Sci Adv. https://doi.org/10.1126/ sciadv. 1603041

Dickinson MH, Götz KG (1996) The wake dynamics and flight forces of the fruit fly Drosophila melanogaster. J Exp Biol 199(9):20852104. https://doi.org/10.1038/35089071

Dickinson MH, Lehmann Fo, Sane SP (1999) Wing rotation and the aerodynamic basis of insect flight. Science 284:1954-1960

Dickson WB, Dickinson MH (2004) The effect of advance ratio on the aerodynamics of revolving wings. J Exp Biol 207(24):4269-81. https://doi.org/10.1242/jeb.01266

Dickson WB, Straw aD, Dickinson MH (2008) Integrative model of Drosophila flight. AIAA J 46(9):2150-2164. https://doi. org/10.2514/1.29862

Dudley R (2002) Mechanisms and implications of animal flight maneuverability. Integr Comp Biol 42(1):135-140. https://doi. org/10.1093/icb/42.1.135

Ellington CP, van den Berg C, Willmott AP, Thomas ALR (1996) Leading-edge vortices in insect flight. Nature 384:626-630

Ennos AR (1989) Inertial and aerodynamic torques on the wings of Diptera in flight. J Exp Biol 142(1):87-95

Fry SN, Sayaman R, Dickinson MH (2003) The aerodynamics of freeflight maneuvers in Drosophila. Science 300(5618):495-498. https ://doi.org/10.1126/science.1081944

Full RJ, Blickhan R, Ting LH (1991) Leg design in hexapedal runners. J Exp Biol 158(1):369-390

Georgiades C, Nahon M, Buehler M (2009) Simulation of an underwater hexapod robot. Ocean Eng 36(1):39-47. https://doi. org/10.1016/j.oceaneng.2008.10.005

Hedrick T, Cheng B, Deng X (2009) Wingbeat time and the scaling of passive rotational damping in flapping flight. Science 324:252-255

Hedrick TL (2008) Software techniques for two- and three-dimensional kinematic measurements of biological and biomimetic systems. Bioinspiration Biomim 3:034001

Hedrick TL, Usherwood JR, Biewener AA (2007) Low speed maneuvering flight of the rose-breasted cockatoo (Eolophus roseicapillus). II. Inertial and aerodynamic reorientation. J Exp Biol 210(11):1912-1924. https://doi.org/10.1242/jeb.002063

Hiley MJ, Yeadon MR (2003) Optimum technique for generating angular momentum in accelerated backward giant circles prior to a dismount. J Appl Biomech 19(2):119-130. https://doi.org/10.1123/ jab.19.2.119

Howe MS (1995) On the force and moment on a body in an incompressible fluid, with application to rigid bodies and bubbles at high and low Reynolds numbers. Q J Mech Appl Math 48(3):401-426. https://doi.org/10.1093/qjmam/48.3.401

Hubel TY, Tropea C (2010) The importance of leading edge vortices under simplified flapping flight conditions at the size scale of birds. J Exp Biol 213(11):1930-1939. https://doi.org/10.1242/ jeb.040857

Ingersoll R, Lentink D (2018) How the hummingbird wingbeat is tuned for efficient hovering. J Exp Biol 221(20):jeb178228. https://doi. org/10.1242/jeb. 178228

Ingersoll R, Haizmann L, Lentink D (2018) Biomechanics of hover performance in Neotropical hummingbirds versus bats. Sci Adv 4(9):eaat2980. https://doi.org/10.1126/sciadv.aat2980

Lammers AR, Zurcher U (2011) Torque around the center of mass: dynamic stability during quadrupedal arboreal locomotion in the Siberian chipmunk (Tamias sibiricus). Zoology 114(2):95-103. https://doi.org/10.1016/j.zool.2010.11.004

Lauder GV (2010) Swimming hydrodynamics: ten questions and the technical approaches needed to resolve them. Springer, Berlin, pp 3-15

Lauder GV, Drucker EG (2003) Morphology and experimental hydrodynamics of piscine control surfaces. Int Symp Unmanned Untethered Submers Technol 29(3):556-571

Lehmann F, Dickinson MH (1998) The control of wing kinematics and flight forces in fruit flies (Drosophila spp.). J Exp Biol 201:385-401

Lehmann FO, Pick S (2007) The aerodynamic benefit of wing-wing interaction depends on stroke trajectory in flapping insect wings. J Exp Biol 210(8):1362-1377. https://doi.org/10.1242/jeb.02746

Lentink D (2018) Accurate fluid force measurement based on control surface integration. Exp Fluids. https://doi.org/10.1007/s0034 8-017-2464-1

Lentink D, Haselsteiner AF, Ingersoll R (2015) In vivo recording of aerodynamic force with an aerodynamic force platform: from drones to birds. J R Soc Interface 12:20141283. https://doi. org/10.1098/rsif.2014.1283

Libby T, Jusufi A, Li D, Cohen DJ, Chang-Siu E, Full RJ, Moore TY (2012) Tail-assisted pitch control in lizards, robots and dinosaurs. Nature 481(7380):181-184. https://doi.org/10.1038/nature10710

Lin T, Zheng L, Hedrick T, Mittal R (2012) The significance of moment-of-inertia variation in flight manoeuvres of butterflies. Bioinspiration Biomim 1:1. https://doi.org/10.1088/17483182/7/4/044002

Magnaudet J (2011) A 'reciprocal' theorem for the prediction of loads on a body moving in an inhomogeneous flow at arbitrary Reynolds number. J Fluid Mech 689:564-604. https://doi.org/10.1017/ jfm.2011.363

Marey E (1890) Physiologie du mouvement: Le vol des oiseaux. G. Masson, Paris

Mitiguy P (2015) Advanced dynamics \& motion simulation. Motion Genesis LLC, Portola Valley

Morouço P, Keskinen KL, Vilas-Boas JP, Fernandes RJ (2011) Relationship between tethered forces and the four swimming techniques performance. J Appl Biomech 27(2):161-169

Park HS, Floyd S, Sitti M (2009) Dynamic modeling and analysis of pitch motion of a basilisk lizard inspired quadruped robot running on water. In: Proceedings-IEEE international conference on robotics and automation, pp 2655-2660. https://doi.org/10.1109/ ROBOT.2009.5152556

Peng J, Dabiri JO (2010) A potential-flow, deformable-body model for fluid-structure interactions with compact vorticity: application to animal swimming measurements. Springer, Berlin, pp 17-26. https://doi.org/10.1007/978-3-642-11633-9

Popovic MB, Goswami A, Herr H (2005) Ground reference points in legged locomotion: definitions, biological trajectories and control implications. Int J Robot Res 24(12):1013-1032. https://doi. org/10.1177/0278364905058363

Protas B (2007) On an attempt to simplify the Quartapelle-Napolitano approach to computation of hydrodynamic forces in open flows. J Fluids Struct 23:1207-1214. https://doi.org/10.1016/j.jfluidstru cts.2007.05.002 
Quartapelle L, Napolitano M (1983) Force and moment in incompressible flows. AIAA J 21(6):911-913. https://doi.org/10.2514/3.8171

Ragazzo CG, Tabak E (2007) On the force and torque on systems of rigid bodies: a remark on an integral formula due to Howe. Phys Fluids 19(5):57108. https://doi.org/10.1063/1.2730481

Ramey MR (1974) The use of angular momentum in the study of longjump take-offs. Macmillan Education, London, pp 144-148. https ://doi.org/10.1007/978-1-349-02612-8-20

Rival DE, van Oudheusden B (2017) Load-estimation techniques for unsteady incompressible flows. Exp Fluids. https://doi. org/10.1007/s00348-017-2304-3

Sardain P, Bessonnet G (2004) Forces acting on a biped robot. Center of pressure-zero moment point. IEEE Trans Syst Man Cybern Part A Syst Hum 34(5):630-637. https://doi.org/10.1109/TSMCA .2004 .832811

Sonin AA (2001) Fundamental Laws of Motion for particles, Material Volumes, and Control Volumes. Massachusetts Institute of Technology, Cambridge, MA

Sugiura H, Dickinson MH (2009) The generation of forces and moments during visual-evoked steering maneuvers in flying Drosophila. PLoS One. https://doi.org/10.1371/journal.pone.0004883

Tan GK, Shen GX, Huang SQ, Su WH, Ke Y (2007) Investigation of flow mechanism of a robotic fish swimming by using flow visualization synchronized with hydrodynamic force measurement. Exp Fluids 43(5):811-821. https://doi.org/10.1007/s00348-007-0407-y

Taylor GK, Thomas AL (2002) Animal flight dynamics II. Longitudinal stability in flapping flight. J Theor Biol 214(3):351-370. https ://doi.org/10.1006/jtbi.2001.2470

Toussaint HM, Vervoorn K (1990) Effects of specific high resistance training in the water on competitive swimmers. Int J Sports Med 11(03):228-233. https://doi.org/10.1055/s-2007-1024797

Triantafyllou MS, Techet AH, Hover FS (2004) Review of experimental work in biomimetic foils. IEEE J Ocean Eng 29(3):585-594. https://doi.org/10.1109/JOE.2004.833216
Tucker VA (2000) Gliding flight: drag and torque of a hawk and a falcon with straight and turned heads, and a lower value for the parasite drag coefficient. J Exp Biol 203(Pt 24):3733-44

Vincenti WG (1982) Control-volume analysis: a difference in thinking between engineering and physics. Technol Cult 23(2):145-174. https://doi.org/10.2307/3104129

Vukobratovic M, Borovac B, Surla D, Stokic D (2012) Biped locomotion: dynamics, stability. Control and application. Communications and control engineering. Springer, Berlin

Winter DA (2009) Biomechanics and motor control of human movement. Wiley, Hoboken, NJ

Woike M, Gewecke M (1978) Kinematic and aerodynamic parameters in tethered flying siskins (Carduelis spinus, Passeres, Aves). J Comp Physiol 127(2):123-129. https://doi.org/10.1007/BF013 52296

Wu JC (1981) Theory for aerodynamic force and moment in viscous flows. AIAA J 19(4):432-441. https://doi.org/10.2514/3.50966

Wu JZ, Pan ZL, Lu XY (2005) Unsteady fluid-dynamic force solely in terms of control-surface integral. Phys Fluids 17(9):98102. https ://doi.org/10.1063/1.2055528

Yates GT (1986) Optimum pitching axes in flapping wing propulsion. J Theor Biol 120(3):255-276. https://doi.org/10.1016/S0022 $-5193(86) 80199-0$

Yeadon MR (1990) The simulation of aerial movement-III. The determination of the angular momentum of the human body. J Biomech 23(1):75-83. https://doi.org/10.1016/0021-9290(90)90371-9

Zatsiorsky VM, Zaciorskij VM (2002) Kinetics of human motion. Human Kinetics, Champaign, IL

Publisher's Note Springer Nature remains neutral with regard to jurisdictional claims in published maps and institutional affiliations. 